\title{
Sensitivity analysis for active control of the Helmholtz equation
}

\author{
M. Hubenthal ${ }^{\mathrm{b}}$, D. Onofrei ${ }^{\mathrm{a}, *}$ \\ ${ }^{a}$ Department of Mathematics, University of Houston, Houston, TX 77004 \\ ${ }^{b}$ Institute for Mathematics and its Applications, University of Minnesota, Minneapolis, MN \\ 55455
}

\begin{abstract}
In previous works we considered the Helmholtz equation with fixed frequency $k$ outside a discrete set of resonant frequencies, where it is implied that, given a source region $D_{a} \subset \mathbb{R}^{d}(d=\overline{2,3})$ and $u_{0}$, a solution of the homogeneous scalar Helmholtz equation in a set containing the control region $D_{c} \subset \mathbb{R}^{d}$, there exists an infinite class of boundary data on $\partial D_{a}$ so that the radiating solution to the corresponding exterior scalar Helmholtz problem in $\mathbb{R}^{d} \backslash D_{a}$ will closely approximate $u_{0}$ in $D_{c}$. Moreover, it will have vanishingly small values beyond a certain large enough "far-field" radius $R$.

In this paper we study the minimal energy solution of the above problem (e.g. the solution obtained by using Tikhonov regularization with the Morozov discrepancy principle) and perform a detailed sensitivity analysis. In this regard we discuss the stability of the minimal energy solution with respect to measurement errors as well as the feasibility of the active scheme (power budget and accuracy) depending on: the mutual distances between the antenna, control region and far field radius $R$; value of the regularization parameter; frequency; location of the source.
\end{abstract}

Keywords: active exterior cloaking, Helmholtz equation, integral equations, regularization

2010 MSC: 45A02, 65R32, 65F 22

\section{Introduction}

The current literature has significantly addressed the idea of active manipulation of vector and scalar fields in desired regions of space.

In fact the active (partial) nulling of fields in the low-frequency acoustics was first studied in [21] (feed-forward control of sound) and in [28] (feedback control of sound). The reviews [33], [15], [19], [20], [32], [9], [31], [7] and references therein discuss the active sound control problem for arbitrary finite frequency.

\footnotetext{
* Corresponding author

Email addresses: huben003@umn.edu (M. Hubenthal), onofrei@math.uh.edu (D. Onofrei)
} 
For cloaking applications, the strategy proposed in [26] employs a continuous active layer on the boundary of the control region while the scheme discussed in $[11,12,13,14,10]$ (see also [37]), uses a discrete number of active sources located in the exterior of the control region to manipulate the fields. These field manipulation problems may be thought of as a prelude to the recommended line of research proposed here. In these works, manipulation of electrostatic fields has been treated as well as time varying scalar fields in two and three dimensions by using integral representation theorems. These results were further extended in [27], where a detailed sensitivity analysis is presented.

In a parallel development in $[34,35]$ the authors make use of the equivalence principle and show how a dipole array can cancel the known electromagnetic scattered field from a cylinder. Further experimental results on active cloaking are presented in [22] for the quasistatic regime and in [6] for the finite frequency regime.

Yet another approach for active cloaking is the use of essentially non-radiating sources. The works [24], [23] and [25] studied the structure of such sources and in $[5,4]$ the author considered their applications for the active cloaking.

In [2] an alternative active scattering cancellation strategy is proposed where it is shown that suitably designed active meta-surfaces (e.g., active smart electrical circuits) can in principle be tuned to suppress the scattering from known incident fields. These results are supported by simulation and in [36] experimental proof was obtained for a cylindrical scatterer of 2.2-wavelengths in length and 0.31-wavelengths in cross section. In all the above works foreknowledge of the fields to be reduced was available.

The active manipulation of quasistatic fields by using one source was studied with the help of boundary layer potentials in [29]. Recently in [30] we extended the results presented in [29] to the active control problem for the exterior scalar Helmholtz equation. In particular, we characterized an infinite class of boundary functions on the source boundary $\partial D_{a}$ so that we achieve the desired manipulation effects in several mutually disjoint exterior regions. The method is novel in the sense that instead of using microstructures, exterior active sources modelled with the help of the above boundary controls are employed for the desired control effects. Such exterior active sources can represent velocity potential, pressure or currents depending on the regime of interest.

In the current paper we propose a sensitivity and feasibility study for the minimal norm solution of the active manipulation problem considered in [29], [30]: one antenna $D_{a}$ approximating a given field in a prescribed near field control region $D_{c}$ with very little radiation on $\partial B_{R}(\mathbf{0})$, with $R \gg 1$ (see Figure 1). We make use of the results in [30] and present a detailed sensitivity and feasibility study for the minimal norm solution of the problem. As we will explain in Section 2 this problem may be relevant to protection from unwanted interrogation as well as for the question of near field synthesis with small far field radiation.

The paper is organized as follows: In Section 2 we present the physical motivation behind our study. In Section 3 we recall the general result obtained in [30] in the context of exterior active cloaking. Section 4 we present an $L^{2}$ 
conditional stability result for the minimal norm solution with respect to measurement errors of the incoming field. In Section 5 we present the numerical details of the Tikhonov regularization algorithm with the Morozov discrepancy principle for the computation of the minimal norm solution of the exterior active cloaking problem in two dimensions. We will numerically observe the fact that the scheme requires large antenna powers in the far field and we will provide numerical support for our theoretical stability results. An important part of this section will be focused on the sensitivity analysis, where we will study: the dependence of the control results as a function of mutual distances between the antenna, control region and far field region; and the broadband character of our scheme in the near field region. Finally, in Section 6 we highlight the main results of the paper and discuss current and future challenges and extensions of our research.

\section{Motivation and main results}

The motivation behind our work is the desire to create stable, low budget schemes, for the approximation of desired fields in the exterior of controlled active sources with possible applications in antenna synthesis, inverse source problems, and electromagnetic or acoustic cloaking/shielding.

Our analysis focuses on a the following situation:

Problem: A single active antenna, $D_{a}$, approximates a desired pattern in its near field region, $D_{c}$, with very little spillover in the far field (e.g. beyond a certain fixed radius $R$ ).

The geometry of the problem is sketched in Figure 1. In Proposition 4.1 and Corollary 4.1 we prove, under suitable source type conditions, the stability of the minimal energy solution (the Morozov solution) of the above problem. In Section 5 we also provide a sensitivity numerical analysis of the minimal energy solution (e.g., its stability, power budget and accuracy) with respect to various parameters: distance between antenna $D_{a}$ and control region $D_{c}$, wave number $k$, noise level $\epsilon$, far field boundary $R$. The numerical sensitivity analysis performed in Section 5 suggest that the scheme proposed by us has a broadband character and is feasible (good accuracy with good power budget) only in a thin sub region in the near field of the sources but possible for a wide enough angular span.

Our result proposes a stable scheme for the synthesis of active sources with controllable near fields and very weak far fields and, besides their possible applicability in near field synthesis applications, this type of sources are theoretically very important in the analysis of the inverse source problems since they are near the kernel of the far field operator and thus are the main cause of instabilities. Their understanding will guide us to a proper penalization of the cost functional to avoid such instabilities. A separate publication with the 3D analysis is in preparation and will be communicated soon.

The presented results imply that one can control a thin but angularly wide near field region of a small active weak radiator and this fact implies the possi- 


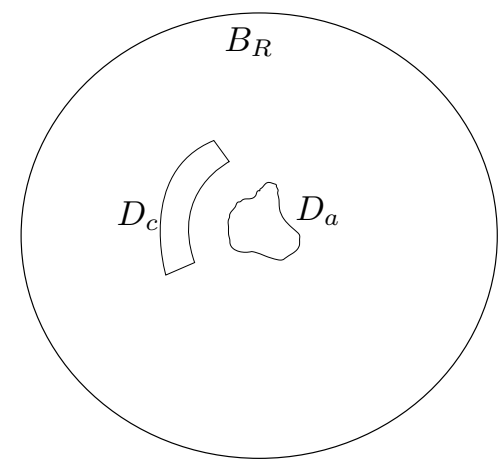

Figure 1: An antenna defined by $\partial D_{a}$ with a control region $D_{c}$ and far field region $B_{R}(\mathbf{0})$.

bility to build a suitable array of such elements with the property that they will cancel an incoming field with very little radiation in the far field. Thus, paired with an appropriate feedback control loop, we believe that such an array could be mounted in a thin electromagenteic transparent skin around a given scatterer (the array being a small distance away from the scatterer) and protect it from unwanted interrogation by cancelling the incident field in the region between the array and the scatterer.

The same question in the acoustic regime is currently studied by our group and will be reported in a forthcoming publication.

\section{Background}

In this section we will recall the main result regarding the active exterior control problem for the Helmholtz equation obtained in [30]. We will focus only on the case where one active external source (antenna) $D_{a}$ protects a control region $D_{c}$ from an interrogating far field and maintains an overall small signature beyond a disk of large enough radius $R$.

The general setup for this question will be as follows. Let $B_{R} \subset \mathbb{R}^{d}$ be the ball of radius $R>0$. We assume $\mathbf{0} \in D_{a} \subset B_{R}$ is the region inside a single antenna with sufficiently smooth boundary $\partial D_{a}$. We also let $D_{c} \Subset B_{R}$ be the control region, which is assumed to satisfy $\overline{D_{c}} \cap \overline{D_{a}}=\emptyset$ (see Figure 1). The numerical simulations in the current work are performed for the two dimensional case but the methods are adaptable to the three dimensional setting as well. Consider the function space

$$
\Xi=L^{2}\left(\partial D_{c}\right) \times L^{2}\left(\partial B_{R}\right)
$$

endowed with the scalar product

$$
(\phi, \psi)_{\Xi}=\int_{\partial D_{c}} \phi_{1}(\mathbf{y}) \bar{\psi}_{1}(\mathbf{y}) d S_{\mathbf{y}}+\int_{\partial B_{R}} \phi_{2}(\mathbf{y}) \bar{\psi}_{2}(\mathbf{y}) d S_{\mathbf{y}}
$$


which is a Hilbert space. For the remainder of the paper we will assume that every $L^{2}$ space of complex valued functions will be endowed with the usual inner product. As in [30] consider $K: L^{2}\left(\partial D_{a}\right) \rightarrow \Xi$, the double layer potential operator restricted to $\partial D_{c}$ and $\partial B_{R}$, respectively, defined by

$$
K \phi(\mathbf{x}, \mathbf{z})=\left(K_{1} \phi(\mathbf{x}), K_{2} \phi(\mathbf{z})\right), \quad \phi \in L^{2}\left(\partial D_{a}\right),
$$

where

$$
\begin{aligned}
& K_{1}: L^{2}\left(\partial D_{a}\right) \rightarrow L^{2}\left(\partial D_{c}\right), K_{1} \phi(\mathbf{x})=\int_{\partial D_{a}} \phi(\mathbf{y}) \frac{\partial \Phi(\mathbf{x}, \mathbf{y})}{\partial \boldsymbol{\nu}_{\mathbf{y}}} d s_{\mathbf{y}}, \text { for } \mathbf{x} \in \partial D_{c} \\
& K_{2}: L^{2}\left(\partial D_{a}\right) \rightarrow L^{2}\left(\partial B_{R}\right), K_{2} \phi(\mathbf{z})=\int_{\partial D_{a}} \phi(\mathbf{y}) \frac{\partial \Phi(\mathbf{z}, \mathbf{y})}{\partial \boldsymbol{\nu}_{\mathbf{y}}} d s_{\mathbf{y}}, \text { for } \mathbf{z} \in \partial B_{R}(\mathbf{0})
\end{aligned}
$$

Here $\Phi(\mathbf{x}, \mathbf{y})$ represents the fundamental solution of the relevant Helmholtz operator, i.e.,

$$
\Phi(\mathbf{x}, \mathbf{y})=\left\{\begin{array}{l}
\frac{e^{i k|\mathbf{x}-\mathbf{y}|}}{4 \pi|\mathbf{x}-\mathbf{y}|}, \text { for } d=3 \\
\frac{i}{4} H_{0}^{(1)}(k|\mathbf{x}-\mathbf{y}|), \text { for } d=2
\end{array}\right.
$$

with $H_{0}^{(1)}=J_{0}+i Y_{0}$ representing the Hankel function of first type. Note that in (3.3) the integrals are to be understood as singular integrals defined through an operator extension from $C\left(\partial D_{a}\right)$. We will also consider $k$ such that

1) $-k^{2}$ is not a Neumann eigenvalue for the Laplace operator in $D_{a}$ or $B_{R}(\mathbf{0})$,

2) $-k^{2}$ is not a Dirichlet eigenvalue for the Laplace operator in $D_{c}$.

As in [30] we introduce the adjoint operator $K^{*}: \Xi \rightarrow L^{2}\left(\partial D_{a}\right)$, which can be shown to satisfy

$$
K^{*} \psi(\mathbf{x})=\int_{\partial D_{c}} \psi_{1}(\mathbf{y}) \frac{\overline{\partial \Phi(\mathbf{y}, \mathbf{x})}}{\partial \nu_{\mathbf{x}}} d S_{\mathbf{y}}+\int_{\partial B_{R}} \psi_{2}(\mathbf{y}) \frac{\overline{\partial \Phi(\mathbf{y}, \mathbf{x})}}{\partial \nu_{\mathbf{x}}} d S_{\mathbf{y}}, \quad \mathbf{x} \in \partial D_{a}
$$

This paper proposes a sensitivity study for the following problem: Let $V \Subset$ $D_{c}$ and $R^{\prime}>R$. For a fixed wave number $k>0$ and fixed $0<\mu \ll 1$, find a function $h \in C\left(\partial D_{a}\right)$ such that there exists $u \in C^{2}\left(\mathbb{R}^{n} \backslash \overline{D_{a}}\right) \cap C^{1}\left(\mathbb{R}^{n} \backslash D_{a}\right)$ solving

$$
\left\{\begin{aligned}
\left(\Delta+k^{2}\right) u(\mathbf{x}) & =0 \quad \mathbf{x} \in \mathbb{R}^{n} \backslash \overline{D_{a}} \\
u & =h \quad \text { on } \partial D_{a} \\
\left\|u-f_{1}\right\|_{C(\bar{V})} & =\mathcal{O}(\mu) \text { and }\|u\|_{C\left(\mathbb{R}^{n} \backslash B_{R^{\prime}}(\mathbf{0})\right)}=\mathcal{O}(\mu),
\end{aligned}\right.
$$

where $f_{1}$ is a solution of the Helmholtz equation in a neighborhood of the control region $D_{c}$. In fact, by using the operator $K$ and regularity arguments it is shown in [30] that a class of solutions for problem (3.7) can be obtained by considering 
the following problem: for a fixed wave number $k>0$ satisfying conditions (3.5), a given function $f=\left(f_{1}, 0\right) \in \Xi$ and $\mu>0$, find a density function $\phi \in C\left(\partial D_{a}\right)$ such that

$$
\|K \phi-f\|_{\Xi} \leq \mu .
$$

Problem (3.8) is in fact a Fredholm integral equation of the first kind, and it was studied in a very general setting in [30]. There the authors proved that the bounded and compact operator $K$ is also one-to-one and has a dense (but not closed) range, thus proving the existence of a class of solutions for (3.8) (and thus for (3.7)). However, the fact that $K$ is compact and that its range is not closed also implies that problem (3.8) is ill-posed. By using regularization, one can approximate a solution to problem (3.8) with an arbitrary level of accuracy $\mu \ll 1$. There are several methods known in the literature, but we will use in this paper the Tikhonov regularization method $[8,1]$. This method, when applied to the operator $K: L^{2}\left(\partial D_{a}\right) \rightarrow \Xi$, proposes a solution $\phi_{\alpha} \in C\left(\partial D_{a}\right)$ of the form

$$
\phi_{\alpha}=\left(\alpha I+K^{*} K\right)^{-1} K^{*} f, \text { for } 0<\alpha \ll 1,
$$

where $\alpha$ is a suitably chosen regularization parameter.

It is known that $\left\|K \phi_{\alpha}-f\right\|_{\Xi} \rightarrow 0$ as $\alpha \rightarrow 0$, (see [17], Theorem 2.16), but the optimal choice of $\alpha$ is an essential step in designing a feasible method (e.g., finding a minimal norm solution), and there are various modalities to do this. In this paper we will use the Morozov discrepancy principle associated to the following weighted residual:

$$
E(\phi, h)=\frac{1}{\left\|h_{1}\right\|_{L^{2}\left(\partial D_{c}\right)}^{2}}\left\|K_{1} \phi-h_{1}\right\|_{L^{2}\left(\partial D_{c}\right)}^{2}+\frac{1}{2 \pi R}\left\|K_{2} \phi\right\|_{L^{2}\left(\partial B_{R}\right)}^{2},
$$

for every given $h=\left(h_{1}, 0\right) \in \Xi$. The reasoning behind using the weighted residual discrepancy functional defined at 3.10 is as follows. Due to the asymptotic behavior of $\frac{\partial \Phi(\mathbf{x}, \mathbf{y})}{\partial \nu_{\mathbf{y}}}=\mathcal{O}\left(|\mathbf{x}-\mathbf{y}|^{-1 / 2}\right)$ as $|\mathbf{x}-\mathbf{y}| \rightarrow \infty$, we have that given a fixed density $\phi,\|K \phi\|_{L^{2}\left(\partial B_{R}\right)}=\mathcal{O}(1)$ as $R \rightarrow \infty$. In other words, using the space $L^{2}\left(\partial B_{R}\right)$ with the standard surface measure is not really suited to the decay properties of double layer potential solutions, because the decay of the normal derivative $\partial_{\nu} \Phi$ is too weak. Similarly, we use the relative norm

$$
\frac{\left\|K_{1} \phi-h_{1}\right\|_{L^{2}\left(\partial D_{c}\right)}}{\left\|h_{1}\right\|_{L^{2}\left(\partial D_{c}\right)}}
$$

on $\partial D_{c}$ because this is a useful quantity for determining how good the control is, regardless of the norm of $h_{1}$. Thus our procedure for finding an approximate solution for problem (3.8) is to first make use of the Tikhonov regularization for the operator $K: L^{2}\left(\partial D_{a}\right) \rightarrow \Xi$ as described in (3.9) to obtain $\phi_{\alpha}$ and then apply the Morozov's discrepancy principle for the choice of $\alpha([18])$, i.e. such that

$$
\begin{array}{r}
E\left(\phi_{\alpha}, f\right)=\delta^{2}, \\
\text { with } \delta^{2} \leq \mu^{2} \min \left\{\frac{1}{2\left\|f_{1}\right\|_{L^{2}\left(\partial D_{c}\right)}^{2}}, \frac{1}{4 \pi R}\right\} .
\end{array}
$$


In what follows, we will account for noise and measurement errors and will consider (3.12) with $f=\left(f_{1}, 0\right) \in \Xi$ replaced by $f_{\epsilon}=\left(f_{\epsilon, 1}, f_{\epsilon, 2}\right) \in \Xi$, given by

$$
f_{\epsilon}=\left(f_{1}+\epsilon s, 0\right) \in \Xi
$$

where $s \in L^{2}\left(\partial D_{c}\right)$ is a random perturbation with $\|s\|_{L^{2}\left(\partial D_{c}\right)} \leq 2\left\|f_{1}\right\|_{L^{2}\left(\partial D_{c}\right)}$ and $f_{1}$ is a solution of the Helmholtz equation in a neighborhood of the control region $D_{c}$. We mention that in the numerical experiments of Section $5, f_{1}$ denotes the $k$ frequency component of the far field of a far field observer. Note that this assumption about the interrogating signal ensures that $f_{1}$ is a solution of the Helmholtz equation in $B_{R}$. In the noisy case (i.e. when $f$ is replaced by $f_{\epsilon}$ ) equation (3.12) becomes

$$
E\left(\phi_{\alpha}, f_{\epsilon}\right)=\delta^{2},
$$

where $\phi_{\alpha}=\left(\alpha I+K^{*} K\right)^{-1} K^{*} f_{\epsilon}$ is the Tikhonov regularization solution. From the definition of $E$ and classical results, [17, 18], it follows that (3.14) admits at least a solution $\alpha$. Moreover, as we will discuss in Section 4, motivated by numerical evidence, we formulate the hypothesis that there exists $\epsilon_{0}>0$ such that for each $\epsilon \in\left(0, \epsilon_{0}\right)$, problem (3.14) has a unique solution $\alpha(\epsilon)$ which uniquely defines a differentiable function $\epsilon \rightsquigarrow \alpha(\epsilon)$. We will study the minimal norm solution uniquely determined by (3.14), discuss its stability for given noisy data in $\Xi$ and, in the case of data corresponding to a point source, analyze its sensitivity with respect to parameters such as: mutual distances between $D_{a}$, $D_{c}$ and $B_{R}(\mathbf{0})$; wave number $k$; and the location of the point source.

\section{Stability estimate for the Tikhonov regularization}

In this section we present analytical and numerical arguments which indicate the stability of the minimum norm solution $\phi_{\alpha}$ with respect to noise level $\epsilon$ for a given fixed discrepancy level $\delta$. Next, we present below Lemma 4.1 which will provide bounds for $\left\|f_{1}\right\|_{L^{2}\left(\partial D_{C}\right)}$ and $\alpha$ in terms of the operatorial norm of $K_{1}^{*}$.

Lemma 4.1. Let $0<\delta<\frac{1}{\sqrt{2}}$ and $z=\left(z_{1}, 0\right) \in \Xi$ with $z_{1} \neq 0$. Consider $v_{\alpha}=\left(\alpha I+K^{*} K\right)^{-1} K^{*} z \in C\left(\partial D_{a}\right)$, with $\alpha$ such that $E\left(v_{\alpha}, z\right) \leq \delta^{2}$. Then we have

$$
\begin{aligned}
\left\|z_{1}\right\|_{L^{2}\left(\partial D_{c}\right)} & \leq 4\left\|K_{1}^{*}\right\|_{\mathcal{O}}\left\|v_{\alpha}\right\|_{L^{2}\left(\partial D_{a}\right)}, \\
\alpha & \leq 4 \delta\left\|K_{1}^{*}\right\|_{\mathcal{O}}^{2},
\end{aligned}
$$

where $K_{1}^{*}$ is the adjoint operator for $K_{1}$ defined by $(3.3)$ and $\|\cdot\|_{\mathcal{O}}$ denotes the operatorial norm.

Proof. We will start with the proof of (4.1). Note that since $E\left(v_{\alpha}, z\right) \leq \delta^{2}$, we have

$$
\left\|K_{1} v_{\alpha}-z_{1}\right\|_{L^{2}\left(\partial D_{c}\right)}^{2} \leq \delta^{2}\left\|z_{1}\right\|_{L^{2}\left(\partial D_{c}\right)}^{2} .
$$


From (4.3) we obtain

$$
\left\|K_{1} v_{\alpha}\right\|_{L^{2}\left(\partial D_{c}\right)}^{2}-2 \operatorname{Re}\left(K_{1} v_{\alpha}, z_{1}\right)_{L^{2}\left(\partial D_{c}\right)}+\left\|z_{1}\right\|_{L^{2}\left(\partial D_{c}\right)}^{2} \leq \delta^{2}\left\|z_{1}\right\|_{L^{2}\left(\partial D_{c}\right)}^{2},
$$

and this implies

$$
\left\|z_{1}\right\|_{L^{2}\left(\partial D_{c}\right)}^{2}\left(1-\delta^{2}\right) \leq 2 \operatorname{Re}\left(v_{\alpha}, K_{1}^{*} z_{1}\right)_{L^{2}\left(\partial D_{a}\right)} \leq 2\left\|v_{\alpha}\right\|_{L^{2}\left(\partial D_{a}\right)}\left\|K_{1}^{*} z_{1}\right\|_{L^{2}\left(\partial D_{a}\right)} .
$$

Then (4.5) gives

$$
\begin{aligned}
\left\|v_{\alpha}\right\|_{L^{2}\left(\partial D_{a}\right)} & \geq \frac{\left\|z_{1}\right\|_{L^{2}\left(\partial D_{c}\right)}^{2}\left(1-\delta^{2}\right)}{2\left\|K_{1}^{*} z_{1}\right\|_{L^{2}\left(\partial D_{a}\right)}}=\left(\frac{1-\delta^{2}}{2}\right)\left(\frac{\left\|z_{1}\right\|_{L^{2}\left(\partial D_{c}\right)}}{\left\|K_{1}^{*} z_{1}\right\|_{L^{2}\left(\partial D_{a}\right)}}\right)\left\|z_{1}\right\|_{L^{2}\left(\partial D_{c}\right)} \\
& \geq \frac{1-\delta^{2}}{2}\left(\frac{\left\|z_{1}\right\|_{L^{2}\left(\partial D_{c}\right)}}{\left\|K_{1}^{*}\right\|_{\mathcal{O}}}\right) \\
& \geq \frac{\left\|z_{1}\right\|_{L^{2}\left(\partial D_{c}\right)}}{4\left\|K_{1}^{*}\right\|_{\mathcal{O}}} .
\end{aligned}
$$

Next we proceed towards proving (4.2). From the definition of $v_{\alpha}$ we have

$$
\begin{aligned}
\alpha v_{\alpha}+K^{*} K v_{\alpha} & =K^{*} z=K_{1}^{*} z_{1}, \\
\alpha v_{\alpha}+K_{2}^{*} K_{2} v_{\alpha} & =K_{1}^{*}\left(z_{1}-K_{1} v_{\alpha}\right) .
\end{aligned}
$$

Here we have used from (3.3) and (3.6) that

$$
\begin{array}{r}
K^{*} \psi=K_{1}^{*} \psi_{1}+K_{2}^{*} \psi_{2}, \text { for all } \psi \in \Xi, \\
K^{*} K w=K_{1}^{*} K_{1} w+K_{2}^{*} K_{2} w, \text { for all } w \in L^{2}\left(\partial D_{a}\right) .
\end{array}
$$

Multiplying (4.7) with $v_{\alpha}$ in the sense of the usual scalar product in $L^{2}\left(\partial D_{a}\right)$, we obtain

$$
\alpha\left\|v_{\alpha}\right\|_{L^{2}\left(\partial D_{a}\right)}^{2}+\left\|K_{2} v_{\alpha}\right\|_{L^{2}\left(\partial D_{a}\right)}^{2}=\left(K_{1}^{*}\left(z_{1}-K_{1} v_{\alpha}\right), v_{\alpha}\right)_{L^{2}\left(\partial D_{a}\right)} .
$$

Using (4.3), (4.6) and (4.8) in (4.9) we then have

$$
\alpha\left\|v_{\alpha}\right\|_{L^{2}\left(\partial D_{a}\right)} \leq \delta\left\|K_{1}^{*}\right\|_{\mathcal{O}}\left\|z_{1}\right\|_{L^{2}\left(\partial D_{c}\right)} \Longrightarrow \alpha \leq 4 \delta\left\|K_{1}^{*}\right\|_{\mathcal{O}}^{2} .
$$

Consider the function $g:(0, \infty) \times(0, \infty) \rightarrow(0, \infty)$ defined by

$$
g(\alpha, \epsilon)=E\left(\phi_{\alpha}, f_{\epsilon}\right),
$$

where $f_{\epsilon} \in \Xi$ was introduced in (3.13), and $\phi_{\alpha}$ is the Tikhonov regularization solution introduced in (3.14). With this notation, (3.14) can be rewritten as

$$
g(\alpha, \epsilon)=\delta^{2},
$$

where $\delta$ is the desired fixed discrepancy level. By using classical results (e.g., $[17,18])$ it can be observed that for every $\epsilon,(4.11)$ admits at least one solution 
in $(0, \infty)$ and that $g$ defined by $(4.10)$ is differentiable with respect to positive $\alpha$ and $\epsilon$, respectively. In fact, it follows from classical arguments that a maximum value of $\alpha$ for a given $\epsilon$ exists. This solution of (4.11) corresponds to the $L^{2}$ minimal energy solution and we will further refer to it as the Morozov solution.

For the remainder of the paper, unless otherwise specified, $C$ will denote a generic constant which depends only on the operator $K, d_{c}=\operatorname{diam}\left(D_{c}\right)$ and $d=\operatorname{dist}\left(\partial D_{c}, \partial D_{a}\right)$. The next Proposition states a central stability result concerning the Morozov solution of (4.11). We have,

Proposition 4.1. Let $0<\delta$ be as above, and $f_{\epsilon}$ and $f_{1}$ as defined in (3.13). For every $\epsilon \geq 0$ consider $\phi_{\alpha_{\epsilon}}=\left(\alpha_{\epsilon} I+K^{*} K\right)^{-1} K^{*} f_{\epsilon} \in C\left(\partial D_{a}\right)$ with $\alpha_{\epsilon}=\alpha(\epsilon)$ the Morozov solution of (4.11). Then we have,

$$
\frac{\left\|\phi_{\alpha_{\epsilon}}-\phi_{\alpha_{0}}\right\|_{L^{2}\left(\partial D_{a}\right)}}{\left\|\phi_{\alpha_{\epsilon}}\right\|_{L^{2}\left(\partial D_{a}\right)}} \leq \frac{\left|\frac{\alpha_{\epsilon}}{\alpha_{0}}-1\right|+\sqrt{\left|\frac{\alpha_{\epsilon}}{\alpha_{0}}-1\right|^{2}+64 \frac{\epsilon(2 \delta+C \delta \epsilon+C \epsilon)}{\alpha_{0}}\left\|K_{1}^{*}\right\|_{\mathcal{O}}^{2}}}{2} .
$$

Proof. Fix $\epsilon>0$ and let $f=f_{\epsilon}$ for $\epsilon=0$. Let us recall that $\alpha(\epsilon)$ is uniquely implicitly defined by the equation $E\left(\left(\alpha_{\epsilon} I+K^{*} K\right)^{-1} K^{*} f_{\epsilon}, f_{\epsilon}\right)=\delta^{2}$ and by Lemma 4.2 it will be differentiable in some interval $\left(0, \epsilon_{0}\right)$ for all wavenumbers $k$. Next consider

$$
\begin{aligned}
\alpha_{\epsilon} \phi_{\alpha_{\epsilon}}+K^{*} K \phi_{\alpha_{\epsilon}} & =K^{*} f_{\epsilon}, \\
\alpha_{0} \phi_{\alpha_{0}}+K^{*} K \phi_{\alpha_{0}} & =K^{*} f .
\end{aligned}
$$

where $\alpha_{0}=\alpha(0), f_{0}=\left(f_{1}, 0\right)$ (as in $\left.(3.13)\right)$ and $\phi_{\alpha_{0}}=\left(\alpha_{0} I+K^{*} K\right)^{-1} K^{*} f_{0}$. Subtracting, we obtain

$$
\begin{aligned}
\alpha_{0} \phi_{\alpha_{0}}-\alpha_{\epsilon} \phi_{\alpha_{\epsilon}}+K^{*} K\left(\phi_{\alpha_{0}}-\phi_{\alpha_{\epsilon}}\right) & =K^{*}\left(f-f_{\epsilon}\right), \\
\alpha_{0}\left(\phi_{\alpha_{0}}-\phi_{\alpha_{\epsilon}}\right)+\left(\alpha_{0}-\alpha_{\epsilon}\right) \phi_{\alpha_{\epsilon}}+K^{*} K\left(\phi_{\alpha_{0}}-\phi_{\alpha_{\epsilon}}\right) & =K^{*}\left(f-f_{\epsilon}\right) .
\end{aligned}
$$

Integrating both sides of (4.13) against $\phi_{\alpha_{0}}-\phi_{\alpha_{\epsilon}}$ yields

$$
\begin{gathered}
\alpha_{0}\left\|\phi_{\alpha_{0}}-\phi_{\alpha_{\epsilon}}\right\|_{L^{2}\left(\partial D_{a}\right)}^{2}+\left(\alpha_{0}-\alpha_{\epsilon}\right)\left(\phi_{\alpha_{\epsilon}}, \phi_{\alpha_{0}}-\phi_{\alpha_{\epsilon}}\right)_{L^{2}\left(\partial D_{a}\right)}+\left\|K\left(\phi_{\alpha_{0}}-\phi_{\alpha_{\epsilon}}\right)\right\|_{\Xi}^{2} \\
=\left(K\left(\phi_{\alpha_{0}}-\phi_{\alpha_{\epsilon}}\right), f-f_{\epsilon}\right)_{\Xi}=\left(K_{1}\left(\phi_{\alpha_{0}}-\phi_{\alpha_{\epsilon}}\right), f_{1}-f_{\epsilon, 1}\right)_{L^{2}\left(\partial D_{c}\right)} .
\end{gathered}
$$

where, we have used (3.3) and the fact that $f_{2}=f_{\epsilon, 2}=0$ in the last equality above. Thus,

$$
\begin{aligned}
\alpha_{0}\left\|\phi_{\alpha_{0}}-\phi_{\alpha_{\epsilon}}\right\|_{L^{2}\left(\partial D_{a}\right)}^{2} & \leq\left|\alpha_{0}-\alpha_{\epsilon}\right|\left\|\phi_{\alpha_{\epsilon}}\right\|_{L^{2}\left(\partial D_{a}\right)}\left\|\phi_{\alpha_{0}}-\phi_{\alpha_{\epsilon}}\right\|_{L^{2}\left(\partial D_{a}\right)} \\
& +\left\|f_{1}-f_{\epsilon, 1}\right\|_{L^{2}\left(\partial D_{c}\right)}\left\|K_{1}\left(\phi_{\alpha_{0}}-\phi_{\alpha_{\epsilon}}\right)\right\|_{L^{2}\left(\partial D_{c}\right)}
\end{aligned}
$$

Observe that

$$
\begin{aligned}
\left\|K_{1}\left(\phi_{\alpha_{0}}-\phi_{\alpha_{\epsilon}}\right)\right\|_{L^{2}\left(\partial D_{c}\right)} & \leq\left\|K_{1} \phi_{\alpha_{0}}-f_{1}\right\|_{L^{2}\left(\partial D_{c}\right)}+\left\|K_{1} \phi_{\alpha_{\epsilon}}-f_{\epsilon, 1}\right\|_{L^{2}\left(\partial D_{c}\right)}+\left\|f_{1}-f_{\epsilon, 1}\right\|_{L^{2}\left(\partial D_{c}\right)} \\
& \leq \delta\left\|f_{1}\right\|_{L^{2}\left(\partial D_{c}\right)}+\delta\left\|f_{\epsilon, 1}\right\|_{L^{2}\left(\partial D_{c}\right)}+C \epsilon\left\|f_{1}\right\|_{L^{2}\left(\partial D_{c}\right)} \\
& =(2 \delta+C \delta \epsilon+C \epsilon)\left\|f_{1}\right\|_{L^{2}\left(\partial D_{c}\right)}
\end{aligned}
$$


where $f_{\epsilon, 1}=f_{1}+\epsilon s$ with $\|s\|_{L^{2}\left(\partial D_{c}\right)} \leq C\left\|f_{1}\right\|_{L^{2}\left(\partial D_{c}\right)}$, and we have used the definition of $\phi_{\alpha_{\epsilon}}$ and (3.10) in the inequalities above. Using (4.16) in (4.15) we obtain

$$
\begin{aligned}
\alpha_{0}\left\|\phi_{\alpha_{0}}-\phi_{\alpha_{\epsilon}}\right\|_{L^{2}\left(\partial D_{a}\right)}^{2} & \leq\left|\alpha_{0}-\alpha_{\epsilon}\right|\left\|\phi_{\alpha_{\epsilon}}\right\|_{L^{2}\left(\partial D_{a}\right)}\left\|\phi_{\alpha_{0}}-\phi_{\alpha_{\epsilon}}\right\|_{L^{2}\left(\partial D_{a}\right)} \\
& +\epsilon(2 \delta+C \delta \epsilon+C \epsilon)\left\|f_{1}\right\|_{L^{2}\left(\partial D_{c}\right)}^{2} .
\end{aligned}
$$

If we define $A:=\frac{\left\|\phi_{\alpha_{\epsilon}}-\phi_{\alpha_{0}}\right\|_{L^{2}\left(\partial D_{a}\right)}}{\left\|\phi_{\alpha_{\epsilon}}\right\|_{L^{2}\left(\partial D_{a}\right)}}$, inequality (4.17) implies that

$$
\begin{aligned}
\alpha_{0} A^{2} & \leq\left|\alpha_{0}-\alpha_{\epsilon}\right| A+\frac{\epsilon(2 \delta+C \delta \epsilon+C \epsilon)\left\|f_{1}\right\|_{L^{2}\left(\partial D_{c}\right)}^{2}}{\left\|\phi_{\alpha_{\epsilon}}\right\|_{L^{2}\left(\partial D_{a}\right)}^{2}} \\
& \leq\left|\alpha_{0}-\alpha_{\epsilon}\right| A+16 \epsilon(2 \delta+C \delta \epsilon+C \epsilon)\left\|K_{1}^{*}\right\|_{\mathcal{O}}^{2},
\end{aligned}
$$

where we have used (4.1) of Lemma 4.1 in the last inequality above. Next, consider

$$
h(A):=A^{2}-\left|\frac{\alpha_{\epsilon}}{\alpha_{0}}-1\right| A-16\left\|K_{1}^{*}\right\|_{\mathcal{O}}^{2} \frac{\epsilon(2 \delta+C \delta \epsilon+C \epsilon)}{\alpha_{0}} .
$$

Then, from (4.18) we have $h(A) \leq 0$ and this implies that

$$
A \leq \frac{\left|\frac{\alpha_{\epsilon}}{\alpha_{0}}-1\right|+\sqrt{\left|\frac{\alpha_{\epsilon}}{\alpha_{0}}-1\right|^{2}+64\left\|K_{1}^{*}\right\|_{\mathcal{O}}^{2} \frac{\epsilon(2 \delta+C \delta \epsilon+C \epsilon)}{\alpha_{0}}}}{2}
$$

which completes the proof.

Next, before presenting the main stability result of this section, we must understand under what conditions equation (4.11) admits a unique solution $\alpha(\epsilon)$ with the property that the resulting function $\epsilon \rightsquigarrow \alpha(\epsilon)$ is differentiable.

We will first discuss the monotonic character of $g$ as this will be essential in establishing the differentiability of $\alpha(\epsilon)$. We first note that, as suggested by the numerics, $g$ is not in general globally monotonic with respect to $\alpha$ as can be seen in Figure 2, which considers an antenna of radius $a=0.01$, region of control characterized in polar coordinates by $r_{1}=0.011, r_{2}=0.015, \theta \in[3 \pi / 4,5 \pi / 4]$, wave number $k=10, f_{\epsilon}$ given by (3.13) with $f_{1}=\frac{1}{4} H_{0}^{(1)}\left(k\left|\mathbf{x}-\mathbf{x}_{0}\right|\right)$ with $\mathbf{x}_{0}=[10000,0]^{T}$, and noise level $\epsilon=0.005$.

On the other hand, for the same geometry and functional settings as in Figure 2, we observe in Figure 3 that for each $\epsilon$ small enough, e.g. $\epsilon<0.015$, $g(\alpha, \epsilon)=E\left(\phi_{\alpha}, f_{\epsilon}\right)$ is strictly increasing with respect to $\alpha$ when $\alpha \in\left(10^{-4}, 1\right)$. Moreover, for every $\epsilon<0.015$ the Morozov solution $\alpha(\epsilon)$ is the unique solution of $(4.11)$ in $\left(10^{-4}, 1\right)$. In other words, for this particular example we have that $\frac{\partial}{\partial \alpha} g(\alpha, \epsilon) \neq 0$ in $\alpha=\alpha_{\epsilon}$ for $\epsilon<0.015$ and this together with the uniqueness and the implicit function theorem implies the differentiability of $\alpha_{\epsilon}$ for $\epsilon<0.015$ !

In fact, numerical results suggest that this conclusion is true for a broadband spectrum of $k$ values. Indeed, let us consider the same geometry and functional 

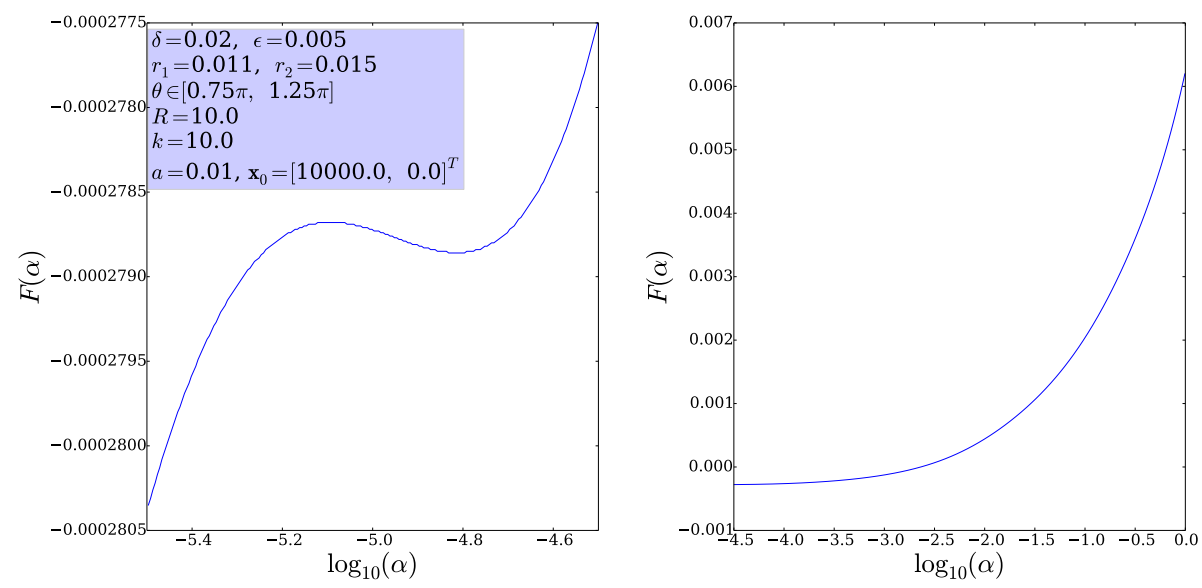

Figure 2: Plot of $F(\alpha)=E\left(\phi_{\alpha}, f_{\epsilon}\right)-\delta^{2}$ for $\epsilon=0.005$ and $\alpha$ in a range where one can see its non-monotonic behavior below a particular threshold.

settings as in Figure 2, Figure 3 with $k \in[1,100]$ and $\epsilon=0.005$. Table 1 summarizes the values of $p_{k}>0$ for which $g(\alpha, \epsilon)$ is locally strictly monotonic with respect to $\alpha$ in an interval $\left(10^{-p_{k}}, 1\right)$, as well as the value of the Morozov solution $\alpha(\epsilon, k)$ for each $k$. We can see from Table 1 and Figure 4 that $\alpha(\epsilon, k) \epsilon$ $\left(10^{-p_{k}}, 1\right)$ and that due to the monotonicity of $g, \alpha(\epsilon, k)$ it is the unique solution of $(4.11)$ in $\left(10^{-p_{k}}, 1\right)$. This also suggests that $\frac{\partial}{\partial \alpha} g(\alpha, \epsilon) \neq 0$ in $\alpha=\alpha(\epsilon, k)$ for small enough $\epsilon$, which again, together with the uniqueness and implicit function theorem, implies the differentiability of $\alpha(\epsilon, k)$ for small enough $\epsilon$ !

For simplicity of notation, in what follows we will write sometimes $\alpha$ instead of $\alpha_{\epsilon}=\alpha(\epsilon)$ and we will use $\alpha^{\prime}$ and $f_{\epsilon, i}^{\prime}$ to denote $\frac{d \alpha}{d \epsilon}$ and $\frac{d f_{\epsilon, i}}{d \epsilon}$ respectively. Motivated by the above numerics, we formulate the following more general hypothesis:

Hypothesis 1. Assume the same geometrical setup as in Section 3 and let $f_{\epsilon}, f_{1}$ be as in (3.13). Then there exists $p_{0}>0$ and $\epsilon_{0}>0$ such that $\frac{\partial}{\partial \alpha} g(\alpha, \epsilon) \neq 0$ in $\left(10^{-p_{0}}, 1\right) \times\left(0, \epsilon_{0}\right)$ for all wave numbers $k$, and the Morozov solution $\alpha(\epsilon)$ is the unique solution of $(4.11)$ in $\left(10^{-p_{0}}, 1\right)$.

For example, as discussed above, for $f_{\epsilon}, f_{1}$ as in (3.13) with $s=\widehat{\nu}\left\|f_{1}\right\|_{L^{2}\left(\partial D_{c}\right)}$ where $\widehat{\nu} \in L^{2}\left(\partial D_{c}\right)$ is a random perturbation with $\|\widehat{\nu}\|_{L^{2}\left(\partial D_{c}\right)}=1$, and for the same geometry and data as in Figure 2 and Figure 3, we have that Hypothesis 1 is satisfied for $p_{0}=10^{-4}$ and $\epsilon$ small enough for all $k=\overline{1,100}$. As a consequence of uniqueness and implicit function theorem we have that whenever Hypothesis 1 is satisfied, the definition of $\alpha(\epsilon)$ and the implicit function theorem imply:

Lemma 4.2. There exists $\epsilon_{0}>0$ such that for every $\epsilon \in\left(0, \epsilon_{0}\right)$, the function $\alpha:\left(0, \epsilon_{0}\right) \rightarrow(0, \infty)$, where for each $\epsilon \in\left(0, \epsilon_{0}\right), \alpha(\epsilon)$ represents the Morozov solution of (4.11), will be differentiable for all wave numbers $k$. 

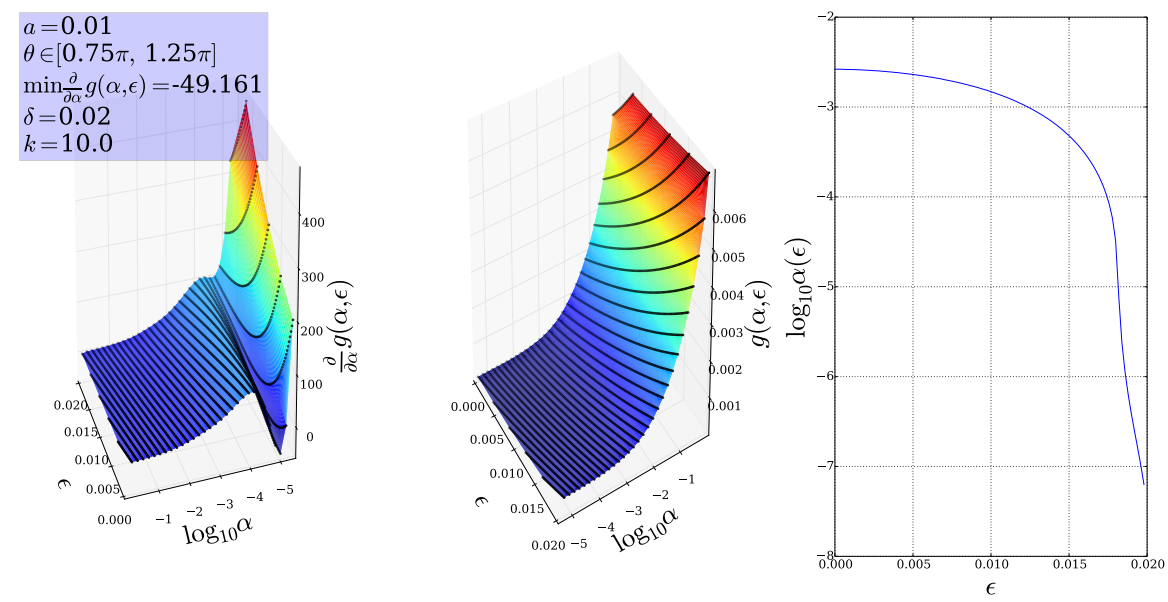

Figure 3: Plot of $g(\alpha, \epsilon)=E\left(\phi_{\alpha}, f_{\epsilon}\right)$ and $\frac{\partial}{\partial \alpha} g(\alpha, \epsilon)$ with respect to $\alpha, \epsilon$ together with the unique largest value $\alpha(\epsilon)$ such that $E\left(\phi_{\alpha_{\epsilon}}, f_{\epsilon}\right)=\delta^{2}$, where $\delta=0.02$ is fixed.

\begin{tabular}{||lll|lll||}
$k$ & $-p_{k}$ & Morozov $\alpha$ & $k$ & $-p_{k}$ & Morozov $\alpha$ \\
\hline \hline 1.0 & -5.74057337341 & 0.0021397 & 51.0 & -5.63387601498 & 0.023987 \\
\hline 6.0 & -5.15371857022 & 0.0022445 & 56.0 & -5.5538513243 & 0.028226 \\
\hline 11.0 & -4.61487654411 & 0.0027985 & 61.0 & -5.58052339557 & 0.032734 \\
\hline 16.0 & -4.43348001737 & 0.0037643 & 66.0 & -7.93866063316 & 0.038053 \\
\hline 21.0 & -7.22374205154 & 0.0052228 & 71.0 & -7.78393971408 & 0.043891 \\
\hline 26.0 & -6.73292218326 & 0.0072707 & 76.0 & -7.60786606025 & 0.048884 \\
\hline 31.0 & -6.41280555768 & 0.0098052 & 81.0 & -7.41580451387 & 0.05425 \\
\hline 36.0 & -6.18339417827 & 0.012607 & 86.0 & -7.2077344143 & 0.060871 \\
\hline 41.0 & -5.97530913764 & 0.015782 & 91.0 & -7.00500135956 & 0.066982 \\
\hline 46.0 & -5.78858575492 & 0.01971 & 96.0 & -6.90364624001 & 0.07278 \\
\hline
\end{tabular}

Table 1: Table of values $-p_{k}$ such that $g(\alpha, \epsilon)$ is increasing with respect to $\alpha$ for $\alpha \geq 10^{-p_{k}}$. $\epsilon$ is fixed at 0.005 in this case. 


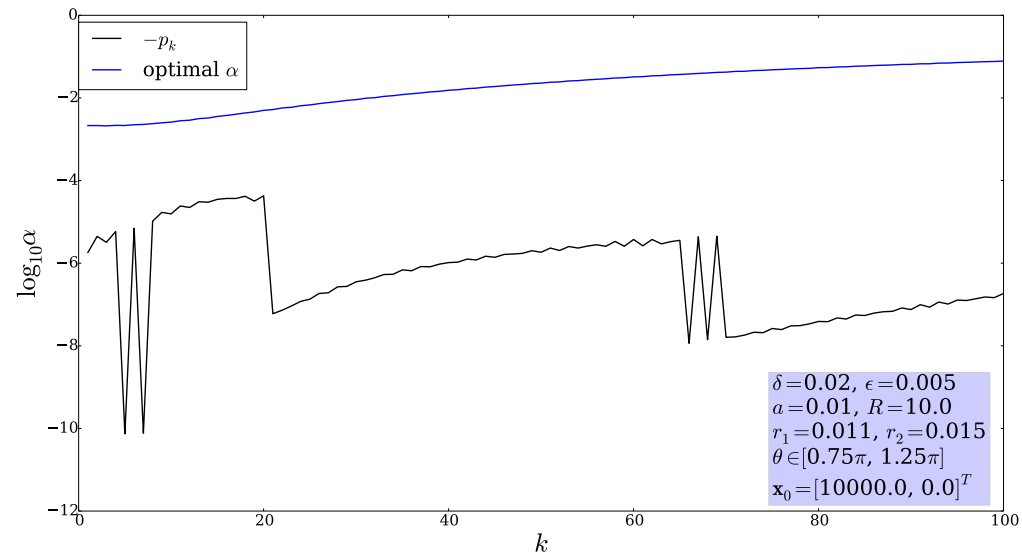

Figure 4: Threshold value $p_{k}$ for which $F(\alpha)$ is increasing when $\alpha>10^{-p_{k}}$. Also shows the value of $\alpha$ for which $F(\alpha)=\delta^{2}$ with the same setting as in Figure 3, where $\delta=0.02$ and the noise level $\epsilon=0.005$.

The next Lemma is a technical result needed in the stability estimate obtained in Corollary 4.1.

Lemma 4.3. Let $f_{1}$ be a solution of the Helmholtz equation in a neighborhood of $D_{c}$ satisfying the following source type condition:

$\left\|K_{1} \psi_{0}-\frac{f_{1}}{\left\|f_{1}\right\|_{L^{2}\left(\partial D_{c}\right)}}\right\|_{L^{2}\left(\partial D_{c}\right)} \leq C \delta$ for some $\psi_{0} \in L^{2}\left(\partial D_{a}\right)$ with $\left\|\psi_{0}\right\|_{L^{2}\left(\partial D_{a}\right)} \leq C \delta$.

Assume $R$ (radius of $B_{R}(\mathbf{0})$ ) is such that,

$$
\left\|f_{1}\right\|_{L^{2}\left(\partial D_{c}\right)} \leq \sqrt{\pi R} .
$$

Consider $s_{\epsilon}=f_{1}+\frac{\epsilon}{2} \widehat{\nu}\left\|f_{1}\right\|_{L^{2}\left(\partial D_{c}\right)}$ where $\widehat{\nu} \in L^{2}\left(\partial D_{c}\right)$ is a random perturbation with $\|\widehat{\nu}\| \leq 1$. Assume the same functional framework as in Proposition 4.1 and that Hypothesis 1 holds true in the case when $f_{\epsilon}$ is given by

$$
f_{\epsilon}=\left(f_{\epsilon, 1}, f_{\epsilon, 2}\right)=\left(f_{1}+\epsilon s_{\epsilon}, 0\right) .
$$

Then, there exists $\epsilon_{0}>0$ such that the Morozov solution of equation (4.11) $\alpha=\alpha(\epsilon)$ satisfies

$$
\alpha\left|\alpha^{\prime}\right| \leq C \frac{\delta^{2}}{\sqrt{\alpha}}, \text { for all } \epsilon<\epsilon_{0} .
$$

Proof. Define the weights

$$
\begin{aligned}
w^{1} & :=\frac{1}{\left\|f_{\epsilon, 1}\right\|_{L^{2}\left(\partial D_{c}\right)}^{2}} \\
w^{2} & :=\frac{1}{2 \pi R} .
\end{aligned}
$$


and denote $T_{\alpha}:=\left(K^{*} K+\alpha I\right)^{-1}, R_{\alpha}:=T_{\alpha} K^{*}$. Then using the Einstein summation convention, we may write

$$
E\left(\phi_{\alpha}, f_{\epsilon}\right)=w^{i}\left\|K_{i} \phi_{\alpha}-f_{\epsilon, i}\right\|_{L^{2}\left(W_{i}\right)}^{2}=w^{i}\left(K_{i} \phi_{\alpha}-f_{\epsilon, i}, K_{i} \phi_{\alpha}-f_{\epsilon, i}\right)_{L^{2}\left(W_{i}\right)},
$$

where $W_{1}=\partial D_{c}, W_{2}=\partial B_{R}$. Next, as in Lemma 4.2, we observe that Hypothesis 1 together with the implicit function theorem imply the uniqueness and differentiability of $\alpha(\epsilon)$, on the interval $\epsilon \in\left(0, \epsilon_{0}\right)$ for some $\epsilon_{0}>0$, where $\alpha(\epsilon)$ is uniquely and implicitly defined by the equation $E\left(\left(\alpha_{\epsilon} I+K^{*} K\right)^{-1} K^{*} f_{\epsilon}, f_{\epsilon}\right)=$ $\delta^{2}$. Differentiating the equation $E\left(\left(\alpha_{\epsilon} I+K^{*} K\right)^{-1} K^{*} f_{\epsilon}, f_{\epsilon}\right)=\delta^{2}$ with respect to $\epsilon$ and noting that $\delta$ is fixed, we obtain

$$
\begin{aligned}
0=\partial_{\epsilon} E\left(\phi_{\alpha}, f_{\epsilon}\right)= & 2 w^{i} \operatorname{Re}\left(K_{i} \phi_{\alpha}^{\prime}-f_{\epsilon, i}^{\prime}, K_{i} \phi_{\alpha}-f_{\epsilon, i}\right)_{L^{2}\left(W_{i}\right)} \\
& -2\left(w^{1}\right)^{2} \operatorname{Re}\left(f_{\epsilon, 1}^{\prime}, f_{\epsilon, 1}\right)_{L^{2}\left(\partial D_{c}\right)}\left\|K_{1} \phi_{\alpha}-f_{\epsilon, 1}\right\|_{L^{2}\left(\partial D_{c}\right)}^{2} .
\end{aligned}
$$

Next, from $\left(K^{*} K+\alpha I\right) \phi_{\alpha}=K^{*} f_{\epsilon}$ we observe that

$$
\phi_{\alpha}^{\prime}=R_{\alpha} f_{\epsilon}^{\prime}-\alpha^{\prime} T_{\alpha} \phi_{\alpha}
$$

Thus, we may write

$$
K_{i} \phi_{\alpha}^{\prime}-f_{\epsilon, i}^{\prime}=-\alpha^{\prime} K_{i} T_{\alpha} \phi_{\alpha}+K_{i} R_{\alpha} f_{\epsilon}^{\prime}-f_{\epsilon, i}^{\prime} .
$$

By using (4.25) and (4.24) we obtain that

$$
\begin{aligned}
2\left(K_{i} \phi_{\alpha}^{\prime}-f_{\epsilon, i}^{\prime}, K_{i} \phi_{\alpha}\right)_{L^{2}\left(W_{i}\right)} & =-2 \alpha^{\prime}\left(T_{\alpha} \phi_{\alpha}, K_{i}^{*} K_{i} \phi_{\alpha}\right)_{L^{2}\left(\partial D_{a}\right)} \\
& +2\left(K_{i} R_{\alpha} f_{\epsilon}^{\prime}-f_{\epsilon, i}^{\prime}, K_{i} \phi_{\alpha}\right)_{L^{2}\left(W_{i}\right)},
\end{aligned}
$$

and

$$
\begin{aligned}
-2\left(f_{\epsilon, i}, K_{i} \phi_{\alpha}^{\prime}-f_{\epsilon, i}^{\prime}\right)_{L^{2}\left(W_{i}\right)} & =2 \alpha^{\prime}\left(f_{\epsilon, i}, K_{i} T_{\alpha} \phi_{\alpha}\right)_{L^{2}\left(W_{i}\right)}-2\left(f_{\epsilon, i}, K_{i} R_{\alpha} f_{\epsilon}^{\prime}-f_{\epsilon, i}^{\prime}\right)_{L^{2}\left(W_{i}\right)} \\
& =2 \alpha^{\prime}\left(K_{i}^{*} f_{\epsilon, i}, T_{\alpha} \phi_{\alpha}\right)_{L^{2}\left(\partial D_{a}\right)}-2\left(f_{\epsilon, i}, K_{i} R_{\alpha} f_{\epsilon}^{\prime}-f_{\epsilon, i}^{\prime}\right)_{L^{2}\left(W_{i}\right)} .
\end{aligned}
$$

Let $P, Q$ be defined by

$$
\begin{aligned}
& P=2 w^{i}\left[\left(K_{i} \phi_{\alpha}-f_{\epsilon, i}, K_{i} R_{\alpha} f_{\epsilon}^{\prime}-f_{\epsilon, i}^{\prime}\right)_{L^{2}\left(W_{i}\right)}+\alpha^{\prime}\left(K_{i}^{*} f_{\epsilon, i}-K_{i}^{*} K_{i} \phi_{\alpha}, T_{\alpha} \phi_{\alpha}\right)_{L^{2}\left(\partial D_{a}\right)}\right] \\
& Q=2\left(w^{1}\right)^{2}\left(f_{\epsilon, 1}^{\prime}, f_{\epsilon, 1}\right)_{L^{2}\left(\partial D_{c}\right)}\left\|K_{1} \phi_{\alpha}-f_{\epsilon, 1}\right\|^{2} .
\end{aligned}
$$

Then from (4.26), (4.27) used in (4.23) we obtain

$$
0=\partial_{\epsilon} E\left(\phi_{\alpha}, f_{\epsilon}\right)=\operatorname{Re}(P)-\operatorname{Re}(Q) .
$$

We focus first on $P$ introduced in (4.28). In this regard, let us define

$$
L_{i}:=\left(K_{i} \phi_{\alpha}-f_{\epsilon, i}, K_{i} R_{\alpha} f_{\epsilon}^{\prime}-f_{\epsilon, i}^{\prime}\right)_{L^{2}\left(W_{i}\right)} .
$$


Observe that (4.21) implies

$$
f_{\epsilon}^{\prime}=\left(f_{\epsilon, 1}^{\prime}, f_{\epsilon, 2}^{\prime}\right)=\left(f_{1}+\epsilon \widehat{\nu}\left\|f_{1}\right\|_{L^{2}\left(\partial D_{c}\right)}, 0\right) .
$$

First note that by using classical arguments based on the singular value decomposition for $K: L^{2}\left(\partial D_{a}\right) \rightarrow \Xi$, one can adapt the results in [17] (Theorem 2.7) and obtain,

$$
\left\|K R_{\alpha} K z-K z\right\|_{\Xi} \leq C \sqrt{\alpha}\|z\|_{L^{2}\left(\partial D_{a}\right)}, \text { for every } z \in L^{2}\left(\partial D_{a}\right) .
$$

Let $f=\left(\frac{f_{1}}{\left\|f_{1}\right\|_{L^{2}\left(\partial D_{c}\right)}}, 0\right)$ and $v=\frac{f_{\epsilon}^{\prime}}{\left\|f_{1}\right\|_{L^{2}\left(\partial D_{c}\right)}}-f$. By using the definition of $E$ and $\Xi$, (3.10), (4.19), (4.20), (4.32), (4.33), Cauchy's inequality and the triangle inequality in (4.31), we obtain

$$
\begin{aligned}
2\left|w^{i} L_{i}\right| & \leq C \delta\left(\sqrt{w_{1}}\left\|K_{1} R_{\alpha} f_{\epsilon}^{\prime}-f_{\epsilon, 1}^{\prime}\right\|_{L^{2}\left(\partial D_{c}\right)}+\sqrt{w_{2}}\left\|K_{2} R_{\alpha} f_{\epsilon}^{\prime}-f_{\epsilon, 2}^{\prime}\right\|_{L^{2}\left(\partial B_{R}(\mathbf{0})\right)}\right) \\
& \leq C \delta\left(\left\|K_{1} R_{\alpha}(v+f)-\left(v_{1}+f_{1}\right)\right\|_{L^{2}\left(\partial D_{c}\right)}+\left\|K_{2} R_{\alpha}(v+f)-\left(v_{2}+f_{2}\right)\right\|_{L^{2}\left(\partial B_{R}(\mathbf{0})\right)}\right) \\
& \leq C \delta\left\|K R_{\alpha}(v+f)-(v+f)\right\|_{\Xi} \\
& \leq C \delta\left(\left\|K R_{\alpha} f-f\right\|_{\Xi}+\left\|K R_{\alpha} v-v\right\|_{\Xi}\right) \\
& \leq C \delta\left(\left\|K R_{\alpha} K \psi_{0}-K \psi_{0}\right\|_{\Xi}+\left\|K R_{\alpha}\left(K \psi_{0}-f\right)\right\|_{\Xi}+\left\|K \psi_{0}-f\right\|_{\Xi}\right)+C \frac{\delta \epsilon}{\sqrt{\alpha}} \\
& \leq C \delta^{2} \sqrt{\alpha}+C \frac{\delta^{2}}{\sqrt{\alpha}}+C \delta^{2}+C \frac{\delta \epsilon}{\sqrt{\alpha}} \\
& \leq C \frac{\delta^{2}}{\sqrt{\alpha}}
\end{aligned}
$$

where Einstein summation convention was used and where, in the second inequality above we make use of (4.20) to obtain $\sqrt{\frac{w_{2}}{w_{1}}} \leq 1$ and $\sqrt{w_{1}}\left\|f_{1}\right\|_{L^{2}\left(\partial D_{c}\right)}<$ $C$ for small enough $\epsilon$, and in the fourth and fifth inequalities above we have used that $\left\|R_{\alpha}\right\|_{\mathcal{O}} \leq \frac{1}{2 \sqrt{\alpha}}$ (e.g. see [17]), and respectively, that $\epsilon<\delta$ and $\psi_{0}$ satisfies the source condition (4.19).

Expanding $P$ defined in (4.28) and using the fact that $f_{\epsilon, 2}=0$ and $\phi_{\alpha}=$ 
$T_{\alpha} K^{*} f_{\epsilon}=T_{\alpha} K_{1}^{*} f_{\epsilon, 1}$, we obtain

$$
\begin{aligned}
P= & \frac{2 \alpha^{\prime}}{\left\|f_{\epsilon, 1}\right\|_{L^{2}\left(\partial D_{c}\right)}^{2}}\left(K_{1}^{*} f_{\epsilon, 1}, T_{\alpha} \phi_{\alpha}\right)_{L^{2}\left(\partial D_{a}\right)}-\frac{2 \alpha^{\prime}}{\left\|f_{\epsilon, 1}\right\|_{L^{2}\left(\partial D_{c}\right)}^{2}}\left(K_{1}^{*} K_{1} \phi_{\alpha}, T_{\alpha} \phi_{\alpha}\right)_{L^{2}\left(\partial D_{a}\right)} \\
& -\frac{2 \alpha^{\prime}}{2 \pi R}\left(K_{2}^{*} K_{2} \phi_{\alpha}, T_{\alpha} \phi_{\alpha}\right)_{L^{2}\left(\partial D_{a}\right)}+2 w^{i} L_{i} \\
= & \frac{2 \alpha^{\prime}}{\left\|f_{\epsilon, 1}\right\|^{2}}\left(T_{\alpha}^{-1} \phi_{\alpha}, T_{\alpha} \phi_{\alpha}\right)_{L^{2}\left(\partial D_{a}\right)}-\frac{2 \alpha^{\prime}}{\left\|f_{\epsilon, 1}\right\|^{2}}\left(K_{1}^{*} K_{1} \phi_{\alpha}, T_{\alpha} \phi_{\alpha}\right)_{L^{2}\left(\partial D_{a}\right)} \\
& -\frac{2 \alpha^{\prime}}{2 \pi R}\left(K_{2}^{*} K_{2} \phi_{\alpha}, T_{\alpha} \phi_{\alpha}\right)_{L^{2}\left(\partial D_{a}\right)}+2 w^{i} L_{i} \\
= & \frac{2 \alpha^{\prime}}{\left\|f_{\epsilon, 1}\right\|^{2}}\left(\left(\alpha I+K_{2}^{*} K_{2}\right) \phi_{\alpha}, T_{\alpha} \phi_{\alpha}\right)_{L^{2}\left(\partial D_{a}\right)}-\frac{2 \alpha^{\prime}}{2 \pi R}\left(K_{2}^{*} K_{2} \phi_{\alpha}, T_{\alpha} \phi_{\alpha}\right)_{L^{2}\left(\partial D_{a}\right)}+2 w^{i} L_{i} \\
= & \frac{2 \alpha \alpha^{\prime}}{\left\|f_{\epsilon, 1}\right\|^{2}}\left(\phi_{\alpha}, T_{\alpha} \phi_{\alpha}\right)_{L^{2}\left(\partial D_{a}\right)}+\frac{\alpha^{\prime}}{\left\|f_{\epsilon, 1}\right\|^{2}} B\left(K_{2}^{*} K_{2} \phi_{\alpha}, T_{\alpha} \phi_{\alpha}\right)_{L^{2}\left(\partial D_{a}\right)}+2 w^{i} L_{i},
\end{aligned}
$$

where $B=2-\frac{\left\|f_{\epsilon, 1}\right\|_{L^{2}\left(\partial D_{c}\right)}^{2}}{\pi R}$ and we have used that $T_{\alpha}^{-1}=\alpha I+K_{1}^{*} K_{1}+K_{2}^{*} K_{2}$ in the third equality above. Observe that (4.20) implies $B \geq 0$. Introduce the notation $\widetilde{K}_{2}:=\sqrt{B} K_{2}$, and denote $v_{\alpha}:=\frac{\phi_{\alpha}}{\left\|f_{\epsilon, 1}\right\|_{L^{2}\left(\partial D_{c}\right)}}$. Then (4.35) becomes

$$
\begin{aligned}
P & =2 \alpha \alpha^{\prime}\left(v_{\alpha}, T_{\alpha} v_{\alpha}\right)_{L^{2}\left(\partial D_{a}\right)}+\alpha^{\prime}\left(\widetilde{K}_{2}^{*} \widetilde{K}_{2} v_{\alpha}, T_{\alpha} v_{\alpha}\right)_{L^{2}\left(\partial D_{a}\right)}+2 w^{i} L_{i} \\
& =\alpha \alpha^{\prime}\left(v_{\alpha}, T_{\alpha} v_{\alpha}\right)_{L^{2}\left(\partial D_{a}\right)}+\alpha^{\prime}\left(\left(\alpha I+\widetilde{K}_{2}^{*} \widetilde{K}_{2}\right) v_{\alpha}, T_{\alpha} v_{\alpha}\right)_{L^{2}\left(\partial D_{a}\right)}+2 w^{i} L_{i} .
\end{aligned}
$$

From [16] (see Section V.3.10), for any self-adjoint linear operator $A: H \rightarrow H$, where $H$ is a given Hilbert space (real or complex), we have that:

$$
0<\gamma=\inf _{\lambda \in \operatorname{Sp}(A)} \lambda \Longrightarrow(A x, x)_{H} \geq \gamma(x, x)_{H},
$$

where $(\cdot, \cdot)$ in $(4.37)$ denotes the usual Hilbert product and where $\operatorname{Sp}(A)$ denotes the real spectrum of the operator $A$. Then, by using (4.37) for the operator $T_{\alpha}$ we obtain

$$
\begin{aligned}
\left(v_{\alpha}, T_{\alpha} v_{\alpha}\right)_{L^{2}\left(\partial D_{a}\right)} & \geq \frac{1}{\alpha+\mu_{1}^{2}}\left\|v_{\alpha}\right\|_{L^{2}\left(\partial D_{a}\right)}^{2} \geq \frac{1}{1+\mu_{1}^{2}}\left\|v_{\alpha}\right\|_{L^{2}\left(\partial D_{a}\right)}^{2} \\
& \geq C\left\|v_{\alpha}\right\|_{L^{2}\left(\partial D_{a}\right)}^{2}
\end{aligned}
$$

where we have used that $\frac{1}{\alpha+\mu_{1}^{2}}=\inf _{\lambda \in \operatorname{Sp}\left(T_{\alpha}\right)} \lambda$ with $\mu_{1}$ denoting the largest singular value of $K$.

Next consider $D_{\alpha}:=\alpha I+\widetilde{K}_{2}^{*} \widetilde{K}_{2}$. Then, because $D_{\alpha}$ and $T_{\alpha}$ are linear, bounded, self-adjoint, invertible and positive definite operators, we have that 
$D_{\alpha} T_{\alpha}$ will also be linear, bounded, self-adjoint, invertible and have strictly positive eigenvalues. Indeed, for any eigenvalue-eigenvector pair $(x, \lambda)$ of $D_{\alpha} T \alpha$ we have

$$
D_{\alpha} T_{\alpha} x=\lambda x \Rightarrow T_{\alpha} x=\lambda D_{\alpha}^{-1} x \Rightarrow \lambda=\frac{\left(T_{\alpha} x, x\right)}{\left(D_{\alpha}^{-1} x, x\right)} \geq 0 .
$$

Observing that $0 \notin \operatorname{Sp}\left(D_{\alpha} T_{\alpha}\right)$ we have the claim, and the positive definiteness of $D_{\alpha} T_{\alpha}$ follows. Thus we have

$$
\left(\left(\alpha I+\widetilde{K}_{2}^{*} \widetilde{K}_{2}\right) v_{\alpha}, T_{\alpha} v_{\alpha}\right)_{L^{2}\left(\partial D_{a}\right)}=\left(D_{\alpha} v_{\alpha}, T_{\alpha} v_{\alpha}\right)_{L^{2}\left(\partial D_{a}\right)} \geq 0
$$

From (4.36), (4.38) and (4.39) used in (4.30), we obtain

$$
\begin{aligned}
\left|2 w^{i} L_{i}\right|+|Q| & \geq\left|\alpha^{\prime}\right|\left|\alpha\left(v_{\alpha}, T_{\alpha} v_{\alpha}\right)_{L^{2}\left(\partial D_{a}\right)}+\left(D_{\alpha} v_{\alpha}, T_{\alpha} v_{\alpha}\right)_{L^{2}\left(\partial D_{a}\right)}\right| \\
& \geq\left|\alpha^{\prime}\right| C \alpha\left\|v_{\alpha}\right\|_{L^{2}\left(\partial D_{a}\right)}^{2} \\
& \geq C \alpha\left|\alpha^{\prime}\right|\left\|v_{\alpha}\right\|_{L^{2}\left(\partial D_{a}\right)}^{2} .
\end{aligned}
$$

From (4.29) and elementary algebraic manipulations we obtain,

$$
|Q| \leq \frac{2\left\|f_{1}\right\|_{L^{2}\left(\partial D_{c}\right)}}{\left\|f_{\epsilon, 1}\right\|_{L^{2}\left(\partial D_{c}\right)}^{4}}\left\|f_{\epsilon, 1}\right\|_{L^{2}\left(\partial D_{c}\right)} \cdot \delta^{2}\left\|f_{\epsilon, 1}\right\|_{L^{2}\left(\partial D_{c}\right)}^{2}=\frac{2\left\|f_{1}\right\|_{L^{2}\left(\partial D_{c}\right)} \delta^{2}}{\left\|f_{\epsilon, 1}\right\|_{L^{2}\left(\partial D_{c}\right)}} \leq C \delta^{2}
$$

Recalling that $v_{\alpha}:=\frac{\phi_{\alpha}}{\left\|f_{\epsilon, 1}\right\|_{L^{2}\left(\partial D_{c}\right)}}$, Lemma 4.1 implies

$$
\left\|v_{\alpha}\right\|_{L^{2}\left(\partial D_{a}\right)} \geq C .
$$

Then from (4.34), (4.41), and (4.42) used in (4.40) we finally obtain the statement of the Lemma:

$$
\alpha\left|\alpha^{\prime}\right| \leq C \delta^{2}+C \frac{\delta^{2}}{\sqrt{\alpha}} \leq C \frac{\delta^{2}}{\sqrt{\alpha}}, \text { for } \epsilon<\epsilon_{0} .
$$

Remark 4.1. Note that $\left\|s_{\epsilon}\right\|_{L^{2}\left(\partial D_{c}\right)} \leq\left(1+\frac{\epsilon}{2}\right)\left\|f_{1}\right\|_{L^{2}\left(\partial D_{c}\right)}$ and thus $f_{\epsilon}$ as defined above satisfies (3.13).

The next result is a simple consequence of Proposition 4.1 and Lemma 4.3. We have,

Corollary 4.1. Assume the same notation and the same framework as in Proposition 4.1. Assume also that for $d_{c}=\operatorname{diam}\left(D_{c}\right)$ small enough there exists $d=\operatorname{dist}\left(\partial D_{c}, \partial D_{a}\right)$ small enough so that $\alpha(0)=\alpha_{0} \approx \delta$. Then we have

$$
\frac{\left\|\phi_{\alpha_{\epsilon}}-\phi_{\alpha_{0}}\right\|_{L^{2}\left(\partial D_{a}\right)}}{\left\|\phi_{\alpha_{\epsilon}}\right\|_{L^{2}\left(\partial D_{a}\right)}} \leq C \sqrt{\epsilon}
$$


Proof. From Lemma 4.3 we obtain that

$$
\left|\alpha_{\epsilon}^{\prime}\right| \alpha_{\epsilon}^{\frac{3}{2}} \leq C \delta^{2} .
$$

Estimate (4.45) and Cauchy's theorem implies that

$$
\left|\frac{\alpha_{\epsilon}^{\frac{5}{2}}}{\alpha_{0}^{\frac{5}{2}}}-1\right|=\frac{5}{2} \epsilon\left|\alpha_{\epsilon_{*}}^{\prime} \alpha_{\epsilon_{*}}^{\frac{3}{2}} \alpha_{0}^{-\frac{5}{2}}\right| \leq C \epsilon \delta^{2} \alpha_{0}^{-\frac{5}{2}} \leq C \sqrt{\epsilon},
$$

where $\epsilon_{*} \in(0, \epsilon)$ and we used that $\epsilon<\delta$. Next, simple algebraic manipulation and (4.46) imply

$$
\left|\frac{\alpha_{\epsilon}}{\alpha_{0}}-1\right| \leq\left|\frac{\alpha_{\epsilon}^{5}}{\alpha_{0}^{5}}-1\right| \leq C \sqrt{\epsilon}\left(\frac{\alpha_{\epsilon}^{\frac{5}{2}}}{\alpha_{0}^{\frac{5}{2}}}+1\right) \leq C \sqrt{\epsilon}(2+C \sqrt{\epsilon}) \leq C \sqrt{\epsilon} .
$$

This together with Proposition 4.1 imply the statement of the Corollary.

Remark 4.2. We note that all the above results can be adapted to three dimensions. The proof follows exactly the same steps by considering the natural extension of the definition of the discrepancy function $E$ in three dimensions.

Remark 4.3. The assumption made in Corollary 4.1 that $\alpha_{0} \approx \delta$ for $d_{c}=$ $\operatorname{diam}\left(D_{c}\right)$ and $\operatorname{dist}\left(\partial D_{c}, \partial D_{a}\right)=d$ small enough is based on (4.2) of Lemma 4.1 and on the numerical results presented in Section 5. In particular, for the same settings as in Figure 2, Figure 5 shows that given small $d_{c}$ for small enough $d$, we have roughly that $10^{-2.5} \leq \alpha_{0} \leq 10^{-1}$. Since $\delta=2 \cdot 10^{-2}$ we have in this case that $\frac{1}{2 \sqrt{5}} \delta \lesssim \alpha_{0} \lesssim 5 \delta$.

Remark 4.4. As suggested by our numerical results in Section 5 we beleive that the constants (denoted by $C$ ) in Proposition 4.1, Lemma 4.3 and Corollary 4.1 will only have small values for $d_{c}=\operatorname{diam}\left(D_{c}\right)$ and $\operatorname{dist}\left(\partial D_{c}, \partial D_{a}\right)=d$ small enough.

\section{Numerics}

In this section we proceed with the numerical study of the minimal norm solution for (3.12) obtained through Tikhonov regularization with the Morozov discrepancy principle for the choice of the regularization parameter in two dimensions. First we focus on the general setup of our numerical approach, and then in Section 5.1 we discuss more specifically the parameters used in our numerical examples. In Sections 5.2 and 5.3 we present numerical data which demonstrates how stably $\phi$ depends on $f$ and various control statistics for a spherical point source. All figures generally display their respective parameters in an offset legend.

For all of the numerical computations done in this section, we discretize the integral operator $K$ via the method of moment collocation. We refer to 


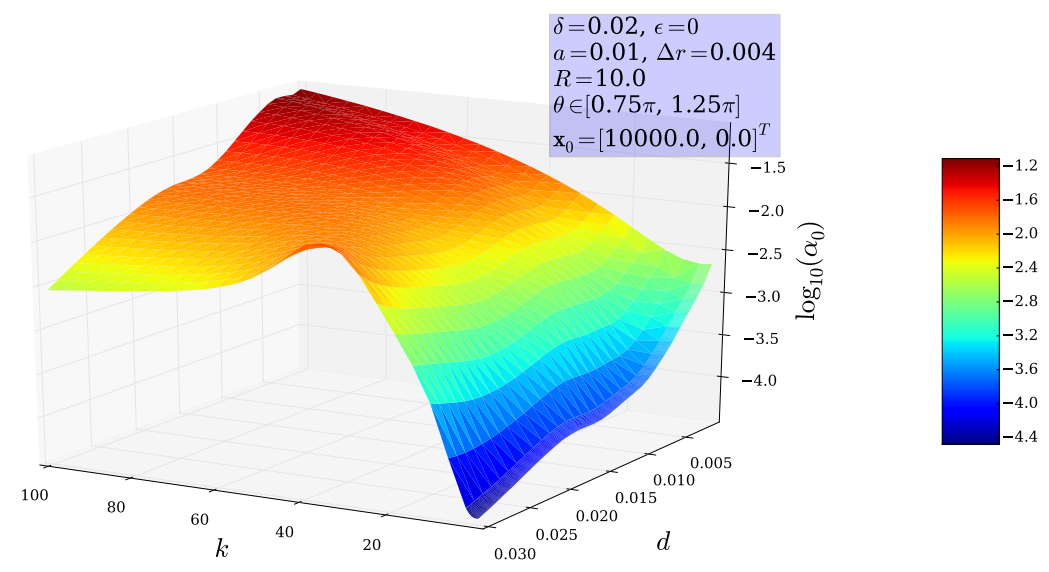

Figure 5: Plot of $\alpha_{0}$ with respect to $k$ and $\mu$ with $\delta=0.02$.

([18], §17.4) for more details on the method. First we choose an approximate basis of functions for $L^{2}\left(\partial D_{a}\right)$. To do this we suppose the domain $D_{a}$ can be parametrized in polar coordinates by points

$$
(s(\tau) \cos \tau, s(\tau) \sin \tau)), \quad \tau \in[0,2 \pi],
$$

where $s: \mathbb{R} \rightarrow \mathbb{R}_{+}$is a $2 \pi$-periodic smooth function. Using these coordinates, any function $\phi$ defined on $\partial D_{a}$ can be realized via the pullback as a function of $\tau$ :

$$
\phi(s(\tau) \cos \tau, s(\tau) \sin \tau) .
$$

For convenience, let us use the notation $\widehat{\tau}=(\cos \tau, \sin \tau)$ and $\widehat{\tau}^{\perp}=(-\sin \tau, \cos \tau)$.

Now let $n_{a} \in \mathbb{N}$ and let $\tau_{j}=\frac{2 \pi j}{n_{a}}, 0 \leq j \leq n_{a}-1$ be $n_{a}$ equally spaced points on the interval $[0,2 \pi)$. We then use the exponential basis functions $\left\{e^{i l \tau}\right\}_{l=0}^{n_{a}-1}$ for $L^{2}([0,2 \pi])$ and approximate a given $\phi \in L^{2}\left(\partial D_{a}\right)$ via interpolation at the points $\left\{\tau_{j}\right\}_{j=0}^{n_{a}-1} \subset[0,2 \pi]$. Note that

$$
\begin{aligned}
\int_{\partial D_{a}} \phi(\mathbf{y}) \frac{\partial \Phi}{\partial \nu_{\mathbf{y}}}(\mathbf{x}, \mathbf{y}) d S_{\mathbf{y}}= & \int_{0}^{2 \pi} \phi(s(\tau) \cos \tau, s(\tau) \sin \tau) \frac{\partial \Phi}{\partial \nu_{y}}(\mathbf{x},(s(\tau) \cos \tau, s(\tau) \sin \tau)) \\
& \cdot \sqrt{s(\tau)^{2}+s^{\prime}(\tau)^{2}} d \theta .
\end{aligned}
$$

Furthermore, since $\left(s^{\prime}(\tau) \cos \tau-s(\tau) \sin \tau, s(\tau) \cos \tau+s^{\prime}(\tau) \sin \tau\right)$ is a tangent vector to $\partial D_{a}$, we have that

$$
\begin{aligned}
\nu(\mathbf{y})=\nu(\tau) & =\frac{\left(s(\tau) \cos \tau+s^{\prime}(\tau) \sin \tau, s(\tau) \sin \tau-s^{\prime}(\tau) \cos \tau\right)}{\sqrt{s(\tau)^{2}+s^{\prime}(\tau)^{2}}} \\
& =\frac{s(\tau) \widehat{\tau}-s^{\prime}(\tau) \widehat{\tau}^{\perp}}{\sqrt{s(\tau)^{2}+s^{\prime}(\tau)^{2}}}
\end{aligned}
$$




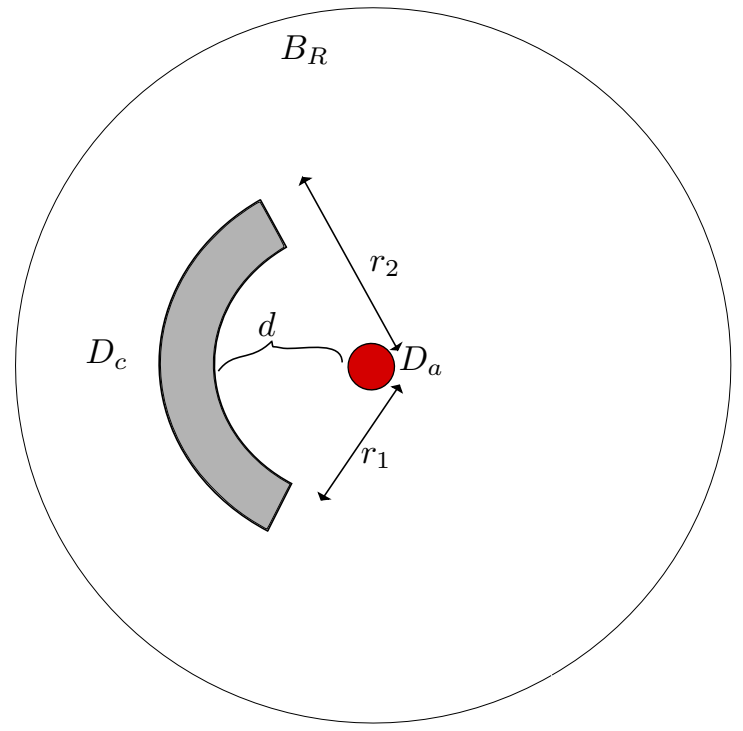

Figure 6: Antenna and control region geometry used for numerical experiments.

is the unit outward normal vector to $\partial D_{a}$. It is then straightforward to compute in the case of the Helmholtz equation in 2D that

$$
\begin{aligned}
& \frac{\partial \Phi}{\partial \nu_{\mathbf{y}}}(\mathbf{x},(s(\tau) \cos \tau, s(\tau) \sin \tau)) \\
= & \nabla_{y} \Phi(\mathbf{x},(s(\tau) \cos \tau, s(\tau) \sin \tau)) \cdot \nu(\tau) \\
= & \frac{i k}{4} H_{0}^{(1)^{\prime}}(k|\mathbf{x}-s(\tau) \widehat{\tau}|) \frac{s(\tau) \widehat{\tau}-\mathbf{x}}{\sqrt{s(\tau)^{2}+|\mathbf{x}|^{2}-2 s(\tau) \mathbf{x} \cdot \widehat{\tau}}} \cdot \frac{s(\tau) \widehat{\tau}-s^{\prime}(\tau) \widehat{\tau}^{\perp}}{\sqrt{s(\tau)^{2}+s^{\prime}(\tau)^{2}}} .
\end{aligned}
$$

Let $n_{a} \in \mathbb{N}$ be the number of sample points on the antenna, $\partial D_{a}$, and let $n_{c} \in \mathbb{N}$ be the total number of sample points on $\partial D_{c}$. Also let $n_{R}$ be the total number of sample points on $\partial B_{R}$. We write the $2 \times\left(n_{c}+n_{R}\right)$ matrix of points

$$
\mathbf{X}:=\left[x_{1}, \ldots, x_{n_{c}+n_{R}}\right],
$$

where each $x_{j}$ is a 2-vector, $\left\{x_{j}\right\}_{j=1}^{n_{c}} \subset \partial D_{c}$ and $\left\{x_{j}\right\}_{j=n_{c}+1}^{n_{c}+n_{R}} \subset \partial B_{R}$. Approximations of all the integrals involved are then computed using a standard left endpoint sum with the appropriate quadrature weights. All the numerical examples presented herein take $D_{c}$ to be an annular sector parametrized by $r \in\left[r_{1}, r_{2}\right]$ and $\theta \in\left[\theta_{1}, \theta_{2}\right]$. See Figure 6 for details.

For each $1 \leq j \leq n_{c}+n_{R}$ and each $0 \leq l \leq n_{a}-1$, we compute $K\left[e^{i l \tau}\right]\left(x_{j}\right)$ via the approximation

$$
\frac{2 \pi}{n_{a}} \sum_{m=0}^{n_{a}-1} \frac{\partial \Phi\left(x_{j},\left[s\left(\tau_{m}\right) \cos \left(\tau_{m}\right), s\left(\tau_{m}\right) \sin \tau_{m}\right]^{T}\right)}{\partial \nu_{\mathbf{y}}} e^{i l \tau_{m}} \sqrt{s\left(\tau_{m}\right)^{2}+s^{\prime}\left(\tau_{m}\right)^{2}} .
$$


If we fix $j$ and vary $l$, we see that the above sum is equivalent to computing the discrete Fourier transform of the $n_{a}$-vector

$$
\mathbf{v}_{j}:=\left[\frac{\partial \Phi\left(x_{j},\left[s\left(\tau_{m}\right) \cos \tau_{m}, s\left(\tau_{m}\right) \sin \tau_{m}\right]^{T}\right)}{\partial \nu_{\mathbf{y}}} \sqrt{s\left(\tau_{m}\right)^{2}+s^{\prime}\left(\tau_{m}\right)^{2}}\right]_{m=0}^{n_{a}-1},
$$

which can be computed efficiently using the Fast Fourier Transform algorithm. In particular, for the vector $\mathbf{v}_{j}$ in (5.2)

$$
\left[K\left\{e^{i l \tau}\right\}\left(x_{j}\right)\right]_{l=0}^{n_{a}-1} \approx 2 \pi \mathrm{FFT}\left(\mathbf{v}_{j}\right),
$$

where FFT is defined on $n_{a}$-vectors $\mathbf{w}=\left[w_{1}, \ldots, w_{n_{a}}\right]^{T} \in \mathbb{C}^{n_{a}}$ by

$$
\operatorname{FFT}(\mathbf{w})=\left[\frac{1}{n_{a}} \sum_{j=1}^{n_{a}} w_{j} e^{\frac{2 \pi i(j-1)(l-1)}{n_{a}}}\right]_{l=1}^{n_{a}} .
$$

So the matrix representation of $K$ is then the $n_{a} \times\left(n_{c}+n_{R}\right)$ matrix

$$
A:=2 \pi\left[\operatorname{FFT}\left(\mathbf{v}_{1}\right), \cdots, \operatorname{FFT}\left(\mathbf{v}_{n_{c}+n_{R}}\right)\right] .
$$

Now, in order to approximately solve the ill-posed problem $K \phi=\left(f_{1}, f_{2}\right)$, we attempt to solve the linear system

$$
\begin{array}{ll}
K_{1} \phi\left(x_{j}\right)=f_{1}\left(x_{j}\right), & 1 \leq j \leq n_{c} \\
K_{2} \phi\left(x_{j}\right)=f_{2}\left(x_{j}\right), & n_{c}+1 \leq j \leq n_{c}+n_{R} .
\end{array}
$$

Since $A$ is computed with respect to the functions $e^{i l \theta}$, we first consider the approximate coefficients of $\phi$ with respect to the finite basis $\left\{e^{i l \tau}\right\}_{l=0}^{n_{a}-1}$, given by

$c_{l}:=\frac{1}{n_{a}} \sum_{m=0}^{n_{a}-1} e^{-i l \tau_{m}} \phi\left(s\left(\tau_{m}\right) \cos \left(\tau_{m}\right), s\left(\tau_{m}\right) \sin \left(\tau_{m}\right)\right) d \tau \approx \frac{1}{2 \pi} \int_{0}^{2 \pi} e^{-i l \tau} \phi(s(\tau) \cos (\tau), s(\tau) \sin (\tau)) d \tau$.

Let

$$
h=\left[c_{0}, c_{1}, \ldots, c_{n_{a}-1}\right]^{T} \in \mathbb{C}^{n_{a}} .
$$

We then numerically compute the Tikhonov regularized solution

$$
h_{\alpha}:=\left(A^{*} A+\alpha I\right)^{-1} A^{*} f,
$$

with $\alpha>0$. The solution vector $h_{\alpha}$ yields the approximate coefficients $c_{l}$ of the desired density $\phi$ with respect to the functions $\left\{e^{i l \tau}\right\}_{l=0}^{n_{a}-1}$. We obtain the density $\phi_{\alpha}$ corresponding to $h_{\alpha}$ sampled at the angles $\tau_{m}$ on $\partial D_{a}$ by the formula

$$
\phi_{\alpha}\left(\tau_{m}\right):=\sum_{l=0}^{n_{a}-1}\left[h_{\alpha}\right]_{l} e^{i l \tau_{m}} .
$$


After computing the residual $K \phi-f$ (e.g. for $\phi=\phi_{\alpha}$ ), we will then need to compute

$$
E\left(\phi_{\alpha}, f\right)=\frac{1}{\left\|f_{1}\right\|_{L^{2}\left(\partial D_{c}\right)}^{2}}\left\|K_{1} \phi-f_{1}\right\|_{L^{2}\left(\partial D_{c}\right)}^{2}+\frac{1}{2 \pi R}\left\|K_{2} \phi-f_{2}\right\|_{L^{2}\left(\partial B_{R}\right)}^{2} .
$$

Recall that the discrepancy function $F(\alpha)$ was defined by

$$
F(\alpha)=E\left(\phi_{\alpha}, f\right)-\delta^{2},
$$

where $\delta>0$ is a fixed error parameter. As discussed in Section 4, the mapping

$$
\alpha \mapsto E\left(\phi_{\alpha}, f\right)
$$

is not globally increasing, as can be numerically demonstrated. However, for certain feasible regions of $(\alpha, \epsilon), F$ is increasing. And in this case, there is a unique $\alpha_{\delta}$ such that $F\left(\alpha_{\delta}\right)=0$. To find $\alpha_{\delta}$, we use Newton's method combined with an initial coarse line search to identify a good starting point.

First note that if we split the matrix $A$ into two blocks $A_{\text {near }}\left(n_{c}\right.$ by $\left.n_{a}\right)$ and $A_{\text {far }}\left(n_{R}\right.$ by $\left.n_{a}\right)$ so that

$$
A=\left[\begin{array}{c}
A_{\text {near }} \\
A_{\text {far }}
\end{array}\right],
$$

then $[A \phi]_{1}=A_{\text {near }} \phi,[A \phi]_{2}=A_{\text {far }} \phi$, and $A^{*} A=A_{\text {near }}^{*} A_{\text {near }}+A_{\text {far }}^{*} A_{\text {far }}$. In the discretized setting, instead of (3.11) we take

$$
F(\alpha)=\frac{1}{\left\|f_{1}\right\|^{2}}\left\|A_{\text {near }} h_{\alpha}-f_{1}\right\|_{L^{2}\left(\partial D_{c}\right)}^{2}+\frac{1}{2 \pi R}\left\|A_{f a r} h_{\alpha}-f_{2}\right\|_{L^{2}\left(\partial B_{R}\right)}^{2}-\delta^{2}
$$

with

$h_{\alpha}=\left(A^{*} A+\alpha I\right)^{-1} A^{*} f=\left(A_{\text {near }}^{*} A_{\text {near }}+A_{\text {far }}^{*} A_{\text {far }}+\alpha I\right)^{-1}\left(A_{\text {near }}^{*} f_{1}+A_{\text {far }}^{*} f_{2}\right)$.

Then in the same spirit as that presented in [3] for Tikhonov regularization with respect to the standard $L^{2}$ norm, we compute

$$
\begin{aligned}
F^{\prime}(\alpha)= & \frac{-2 \alpha}{\left\|f_{1}\right\|_{L^{2}\left(\partial D_{c}\right)}^{2}} \operatorname{Re}\left(\frac{\partial h_{\alpha}}{\partial \alpha}, h_{\alpha}\right) \\
& +\left(\frac{1}{\pi R}-\frac{2}{\left\|f_{1}\right\|_{L^{2}\left(\partial D_{c}\right)}^{2}}\right) \operatorname{Re}\left(\frac{\partial h_{\alpha}}{\partial \alpha}, A_{f a r}^{*} A_{f a r} h_{\alpha}-A_{f a r}^{*} f_{2}\right) \\
\frac{\partial h_{\alpha}}{\partial \alpha}= & -\left(A^{*} A+\alpha I\right)^{-1} h_{\alpha},
\end{aligned}
$$

where $(\cdot, \cdot)$ denotes the $L^{2}$ inner product on $\partial D_{a}$.

The function $f_{1}$ defined on $\partial D_{c}$ could be, for example, the trace of a plane wave, or of the fundamental solution to the Helmholtz equation based at some fixed point $\mathbf{x}_{0}$, i.e. a point source. For this paper, we focus on the case where 
$f_{1}$ is a point source and where $f_{2} \equiv 0$ on $\partial B_{R}$. A spherical point source is represented as

$$
\frac{i}{4} H_{0}^{(1)}\left(k\left|\mathbf{x}-\mathbf{x}_{0}\right|\right),
$$

where $\mathbf{x}_{0}$ is the source point (typically outside of $B_{R}$ ).

For such an $f_{1}$, there are some quantities in which we will be interested so as to determine the effectiveness of a given density $\phi$ in solving the problem $K \phi=f$. These are: the relative error of $K \phi$ on $\partial D_{c}$ which will be indicated by the behavior of $F(\alpha)$; the $L^{2}$ average of $K \phi$ on $\partial B_{R}$; the relative and absolute stability of $\phi$ when applying a small perturbation to $f_{1}$; the norm of $\phi$ on $\partial D_{a}$. More explicitly, we will measure

$$
\begin{gathered}
\sqrt{\frac{F\left(\alpha_{\epsilon}\right)}{\delta}}, \quad \frac{1}{\sqrt{2 \pi R}}\left\|K_{2} \phi_{\alpha_{\epsilon}}\right\|_{L^{2}\left(\partial B_{R}\right)}, \\
\frac{\left\|\phi_{\alpha_{\epsilon}}-\phi_{\alpha_{0}}\right\|_{L^{2}\left(\partial D_{a}\right)}}{\left\|\phi_{\alpha_{0}}\right\|_{L^{2}\left(\partial D_{a}\right)}}, \quad\left\|\phi_{\alpha_{\epsilon}}-\phi_{\alpha_{0}}\right\|_{L^{2}\left(\partial D_{a}\right)},
\end{gathered}
$$

and

$$
\left\|\phi_{\alpha_{\epsilon}}\right\|_{L^{2}\left(\partial D_{a}\right)},
$$

where $\phi_{\alpha_{\epsilon}}$ is the Tikhonov regularization solution to $K \phi=\left(f_{1, \epsilon}, 0\right)$ with $\| f_{1}-$ $f_{1, \epsilon}\left\|_{L^{2}\left(\partial D_{c}\right)}=\epsilon\right\| f_{1} \|_{L^{2}\left(\partial D_{c}\right)}$, and $\phi_{\alpha_{0}}$ is the solution with unperturbed $f_{1}$ with $\alpha_{\epsilon}$ and $\alpha_{0}$ chosen according to the Morozov principle. The Morozov solution $\alpha_{0}$ and $\alpha_{\epsilon}$ are computed via Newton's Method using (5.9) and (5.10) such that

$$
\begin{gathered}
E\left(\phi_{\alpha_{0}}, f\right)=\delta^{2} \\
E\left(\phi_{\alpha_{\epsilon}}, f_{\epsilon}\right)=\delta^{2} .
\end{gathered}
$$

See also the discussion following (5.6). Recall from (3.13), that when adding noise to the data $\left(f_{1}, 0\right)$, we choose a random perturbation $\eta \in L^{2}\left(\partial D_{c}\right)$ and set

$$
f_{1, \epsilon}=f_{1}+\epsilon \widehat{\eta}\left\|f_{1}\right\|_{L^{2}\left(\partial D_{c}\right)},
$$

where $\epsilon>0$ represents the relative percentage of noise added. In the discrete case, the noise is chosen to be a complex $n_{c}$-vector $\nu$ whose real and imaginary components are pseudorandom numbers (we used uniformly distributed noise, but any distribution would yield similar results) on the interval $(-1,1)$. Furthermore, for reproducibility, whenever generating $\nu$ using a pseudorandom number generator, we always reset the seed to the same value.

\subsection{Parameters used for numerical experiments}

Here we describe some of the parameters used for the various numerical experiments presented. In Section 5.3 we always assume that $\partial D_{a}$ is a circle with radius given by $a=0.01$, and that $\partial D_{c}$ is a sector of an annulus with $\theta_{1}=3 \pi / 4$ and $\theta_{2}=5 \pi / 4$. We also take $R=10$ in all computational examples. We remark that we always restrict the distance from $D_{c}$ to $D_{a}$ to be no smaller 
than $10^{-3}$ due to the numerical limitations of our approach. This is due to the fact that $K \phi$ is a singular integral when evaluating at points very near to $\partial D_{a}$. Therefore, at points on $\partial D_{c}$ that are near $\partial D_{a}$ the limitations of machine precision become more and more apparent. Numerically, we observed that our direct approach starts to break down near $d=\operatorname{dist}\left(\partial D_{c}, \partial D_{a}\right)=10^{-4}$. However, we stress that one could most likely obtain high accuracy in computing $K \phi$ for $d \leq 10^{-4}$ by using the Nyström method as discussed in [18].

For the collocation method, we use $n_{a}=256$ sample points on $\partial D_{a}$, and $n_{\operatorname{arc}_{1}}=256$ points on the inner arc of $\partial D_{c}$, with the remaining points chosen so as to keep the quadrature weights approximately constant. Thus for a very thin region, $n_{c} \approx 512$. We also take $n_{R}=256$ (number of sample points on $\left.\partial B_{R}\right)$. Note that increasing $n_{c}$ or $n_{R}$ will put more emphasis on matching $f$ on $\partial D_{c}$ or $\partial B_{R}$, respectively. The discrepancy parameter $\delta$ used for Tikhonov regularization will typically be fixed at 0.02 . The key variables under consideration are $d=r_{1}-a, k$, and $\epsilon$ (perturbation parameter for adding noise to $f_{1}$ ). All of the plots presented in the following sections involve varying two of the aforementioned parameters and plotting different quantities of interest, as stated in (5.12)-(5.14).

When evaluating the relative change in $\phi$ given a perturbation of $f_{1}$, denoted by $f_{\epsilon, 1}$, we remark that for the parameter choices we used, a $0.5 \%$ change $(\epsilon=0.005)$ in $f_{1}$ yielded a roughly $5 \%$ change in $\phi$. However, one must keep in mind that this depends quite a lot on the parameters used. In particular, setting the discrepancy $\delta=0.02$ in all the simulations had an important effect on the numerical results. If we had used $\delta=0.05$ instead (which leads to approximately a $5 \%$ mismatch on the region $\partial D_{c}$ ), then the relative change in $\phi$ given $\epsilon=0.005$ would be noticeably smaller. So ultimately there is a tradeoff between $\mu, R, k, \delta$, and $\epsilon$ which is not entirely trivial, but this still can be examined experimentally as we have done.

\subsection{Near field stability}

We present below Figure 7, which shows how the first 50 singular values of the operator $K=\left(K_{1}, K_{2}\right)$ vary with $d$. It is clear that for $d$ small (i.e. for control in the nearfield of the antenna), the rate of decay of the singular values of $K$ is considerably slower than for larger $d$. This in turn provides some experimental evidence for the fact that nearfield control seems to be more feasible in terms of stable dependence of the solution $\phi$ on $f$. We also show Figure 8, which shows the behavior of the first and sixth singular value of $K$ with respect to $d$ and $k$.

\subsection{Control for a spherical point source}

We now consider the case that

$$
f_{1}(\mathbf{x})=\frac{i}{4} H_{0}^{(1)}\left(k\left|\mathbf{x}-\mathbf{x}_{0}\right|\right),
$$

where $\mathbf{x}_{0}$ is the source point. In all examples presented in this section, we will show plots of the relevant quantities introduced at (5.13), (5.14) and we 


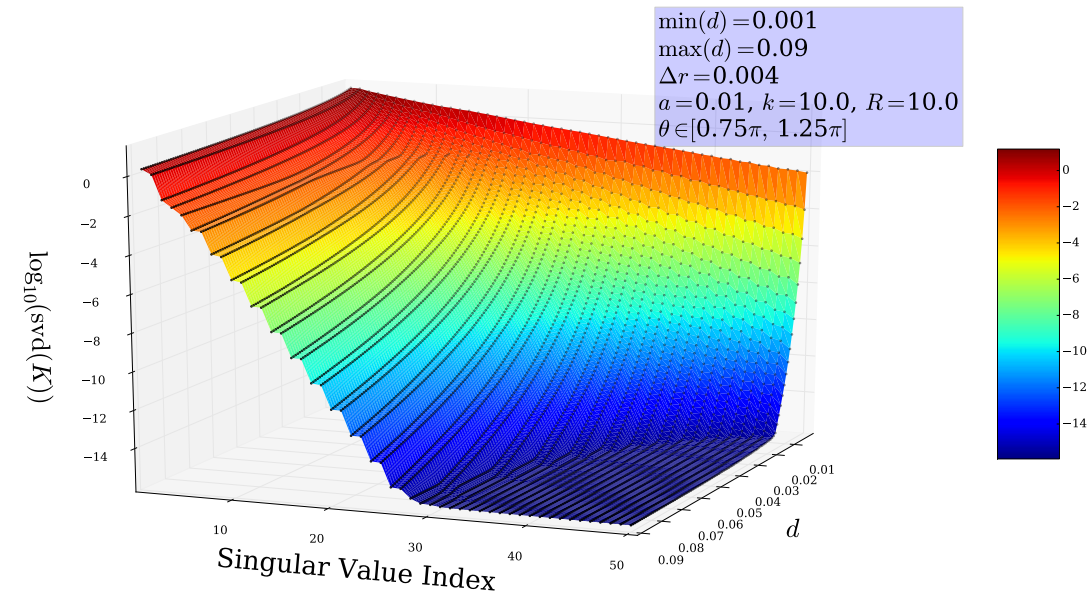

Figure 7: Plot of first 50 singular values of $K: L^{2}\left(\partial D_{a}\right) \rightarrow \Xi$ for $\partial D_{a}$ a circular antenna of radius $a=0.01$ and $\partial D_{c}$ an annular region of varying distance from $\partial D_{a}$.
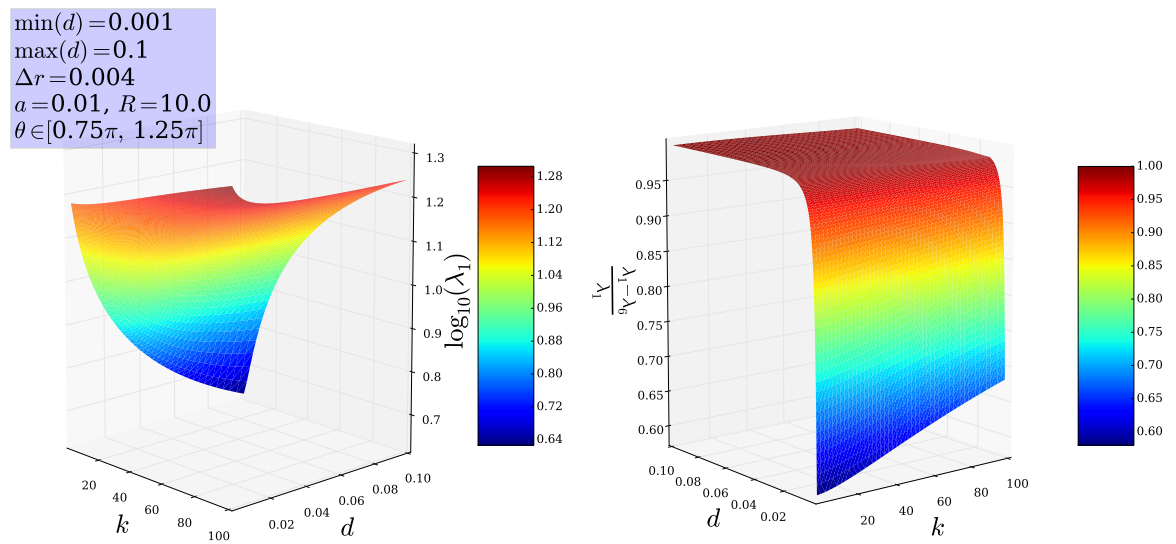

Figure 8: Plot of first singular value of $K: L^{2}\left(\partial D_{a}\right) \rightarrow \Xi$ as well as the relative difference of the first and sixth singular values with respect to $d$ and $k$. Again, $\partial D_{a}$ is a circular antenna of radius $a=0.01$ and $\partial D_{c}$ an annular region. 
assume $R=10$ unless otherwise noted, and $\mathbf{x}_{0}=[20,0]^{T}$ or $\mathbf{x}_{0}=[10000,0]^{T}$ (to approximate a source at infinity).

Figures 9,10 show the plots for the case when $f_{1}$ is a point source located at $(10,000,0)$ and Figure 11, 12 present the similar plots for the case when $f_{1}$ is a point source located at $(20,0)$. We observe how the frequency $k$ and distance $d$ from $\partial D_{c}$ to $\partial D_{a}$ affects the various control criteria. In the figures we vary $k$ from 0.1 to 100 and $d$ from 0.003 to 0.04 with $a=0.01$. With the error discrepancy set at $\delta=0.02$, we have that the relative error on $\partial D_{c}$ is very close to $2 \%$ for all data points. Moreover, with $0.5 \%$ noise added to $f$, roughly a $5 \%$ change in $\phi$ is observed at all frequencies when $d$ is near its lower limit. A bit more sensitivity is observed for frequencies $k<20$ when $d$ increases beyond 0.01 . We observe tough that the power budget $\left(\left\|\phi_{\alpha_{\epsilon}}\right\|_{L^{2}\left(\partial D_{a}\right)}\right)$ is very small for the accurate control and interestingly, for $k>20$ the optimal parameter $\alpha$ is larger and corresponding power budget smaller in order to achieve discrepancy $\delta$.

Figures 10 and 12 support the claim that our sources are indeed weak radiators beyond the radius $R$.

It is clear from the figures that for smaller $d$ values the sensitivity of $\phi$ to $0.5 \%$ noise added to $f$ is close to $5 \%$. As $d$ increases, sensitivity of $\phi$ to noise increases as expected. Having $\mathbf{x}_{0}$ nearer or farther from $\partial B_{R}$ does not have a very significant effect on the overall shape of each subplot.
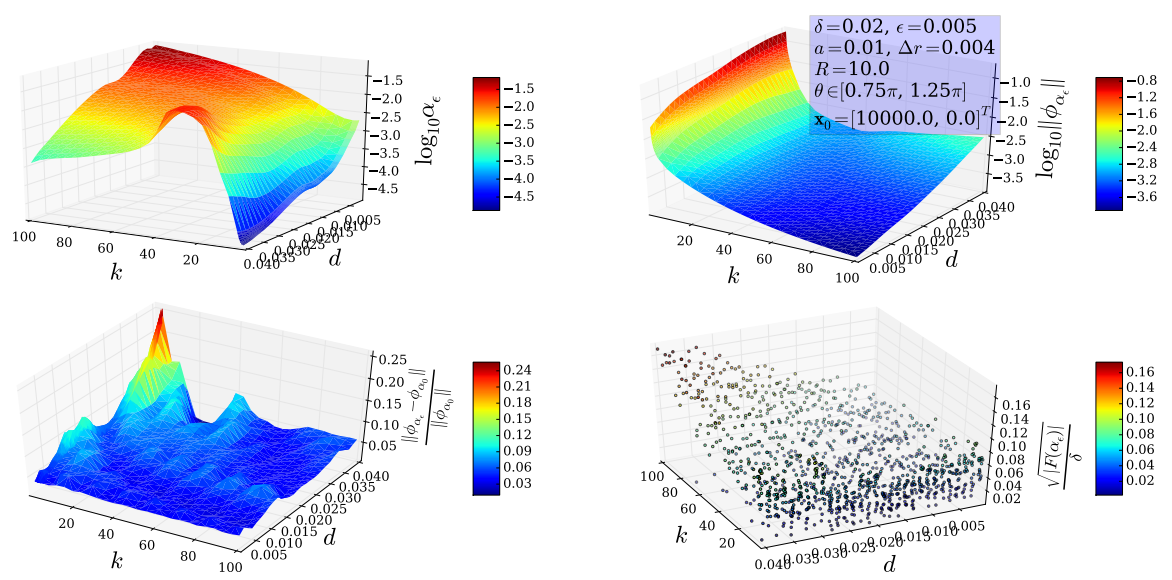

Figure 9: Plot vs. $k$ and $d$ for $f_{1}$ a spherical point source at $\mathbf{x}_{0}=[10000,0]^{T}$.

Figures 13,14 show the plots for the case when $f_{1}$ is a point source located at $(10,000,0)$ and Figures 15,16 present the similar plots for the case when $f_{1}$ is a point source located at $(20,0)$. The numerics indicate how the quantities of interest change with $d$ and the noise factor $\epsilon$, both with $k=10$. The reason for choosing $k=10$ instead of, e.g., $k=1$ is that from figure 9 we see a slightly higher sensitivity of $\phi$ to noise for approximately $1<k<20$ when $d$ starts 


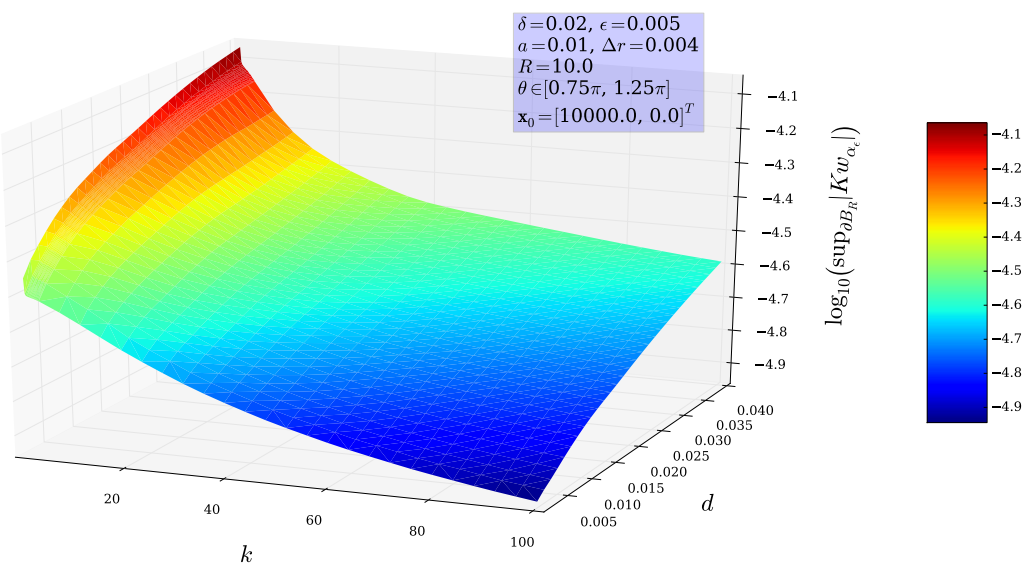

Figure 10: Plot of the far field on $\partial B_{R}$ vs. $d$ and $\epsilon$ for $f_{1}$ a spherical point source at $\mathbf{x}_{0}=[10000,0]^{T}$.
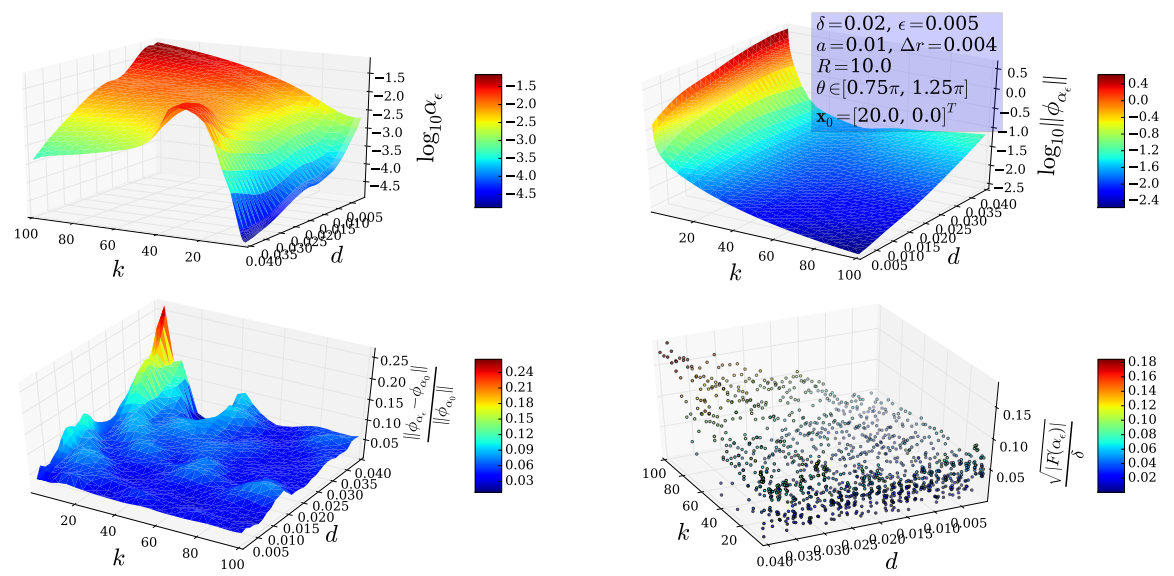

Figure 11: Plot vs. $k$ and $d$ for $f_{1}$ a spherical point source at $\mathbf{x}_{0}=[20,0]^{T}$.

to increase. So the goal was to capture the worst case scenario for the control stability.

For smaller values of $d$ we see as before that a roughly $0.5 \%$ change in $f_{1}$ yields about a $5 \%$ change in $\phi$. Moreover, the dependence on $\epsilon$ for fixed $d$ is superlinear, consistent with the illposedness of the problem. Interestingly, sensitivity of $\phi$ at $d \approx 0.015$ is better than at nearby values, but of course such a value depends on the other parameters of the problem setup. As before, it can be seen in the figures that in both situations our antennas are weak radiators.

Finally, we consider Figure 17, which shows the dependence on $R$ and $k$ for a source at $\mathbf{x}_{0}=[10000,0]^{T}$. Overall, one can see that $R$ can be decreased to around $R=3$ at any frequency between 0.1 and 100 and still achieve the same 


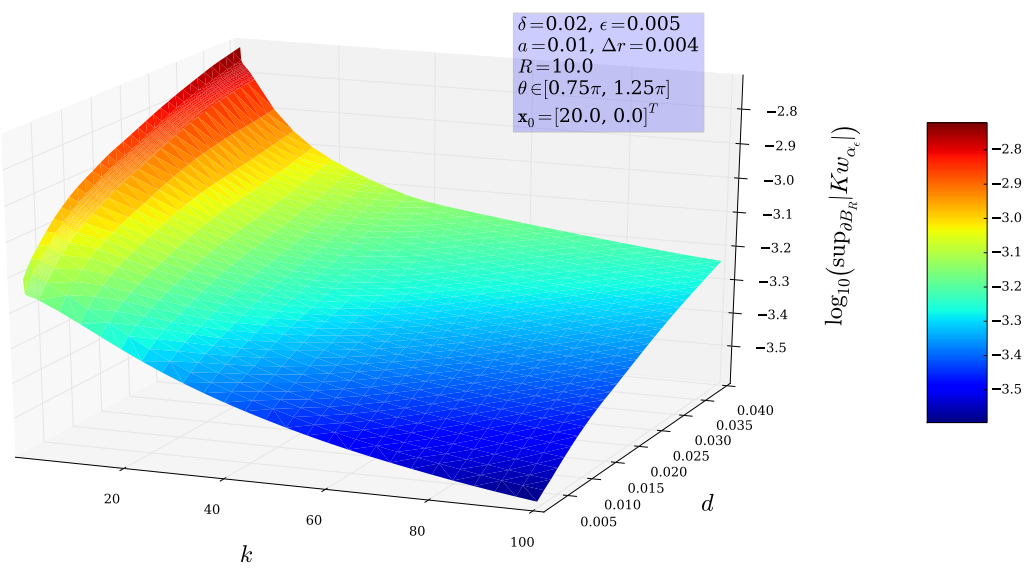

Figure 12: Plot of the far field on $\partial B_{R}$ vs. $k$ and $d$ for $f_{1}$ a spherical point source at $\mathbf{x}_{0}=[20,0]^{T}$.
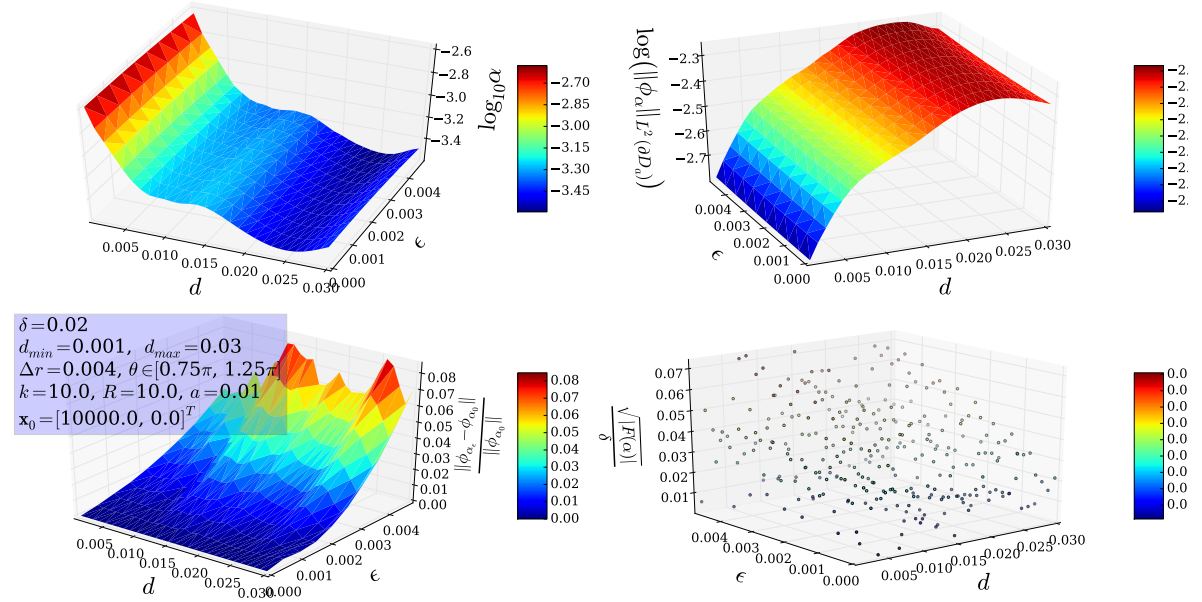

Figure 13: Plot vs. $d$ and $\epsilon$ for $f_{1}$ a spherical point source at $\mathbf{x}_{0}=[10000,0]^{T}$.

approximate level of sensitivity for $\phi$ as in the previous plots with $R=10$.

\section{Conclusions and future work}

In this paper we studied the feasibility of the active control scheme for the scalar Helmholtz equation. In the $L^{2}$ setting, we presented analytic conditional stability results as well as detailed 2D numerical sensitivity studies for the minimal energy solution. We provided analytic and 2D numerical arguments for the scheme's feasibility and broadband character in the near field when the field to be approximated corresponds to an external point source. In Section 2 we highlighted the possible applications of our results for near field synthesis, for 


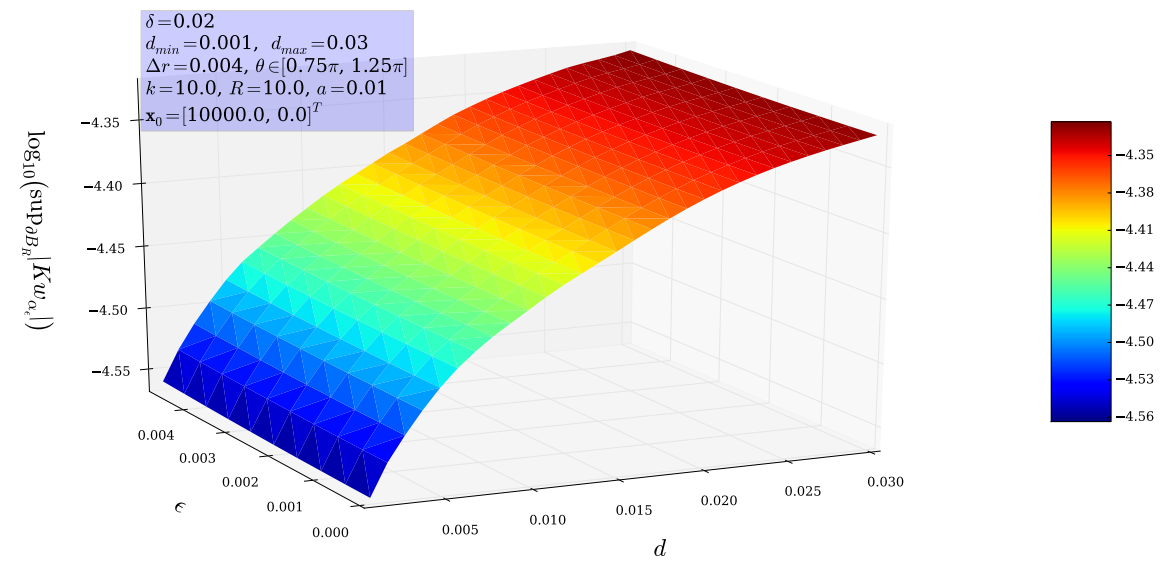

Figure 14: Plot of the far field on $\partial B_{R}$ vs. $d$ and $\epsilon$ for $f_{1}$ a spherical point source at $\mathbf{x}_{0}=[10000,0]^{T}$.
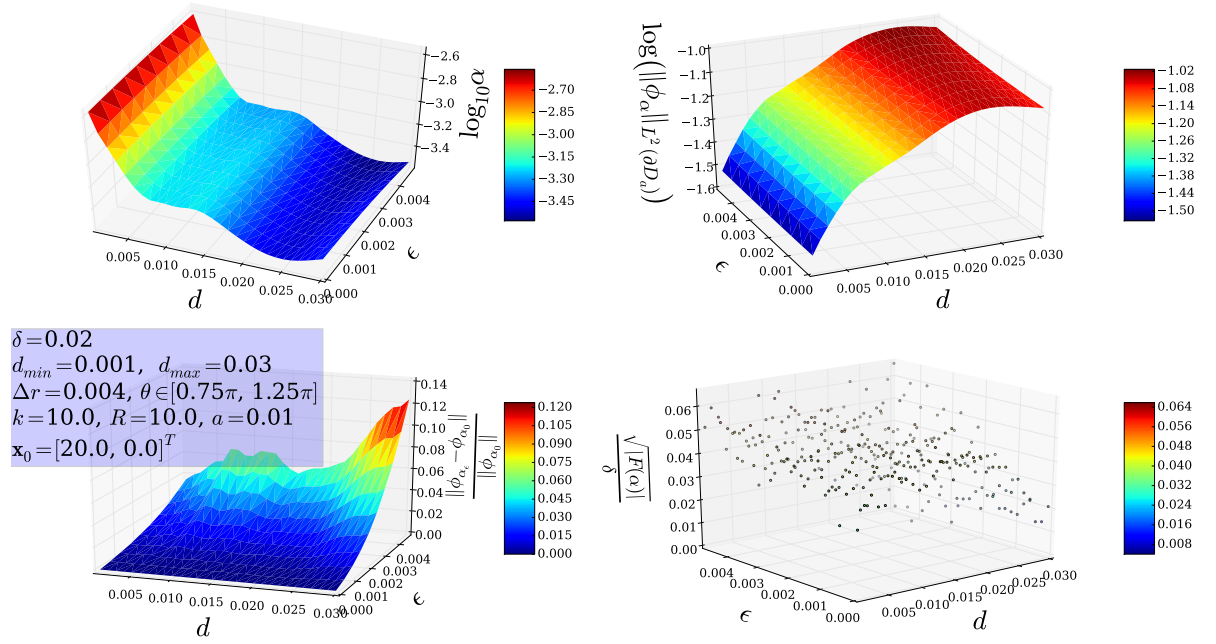

Figure 15: Plot vs. $d$ and $\epsilon$ for $f_{1}$ a spherical point source at $\mathbf{x}_{0}=[20,0]^{T}$.

the characterization of nonradiating sources with controllable near fields and for Radar Cross-Section (RCS) reduction.

We focused our discussion in this paper only on the case of the field to be approximated corresponding to a far field point source (i.e. similar to a plane wave with corresponding decay).

Although we did not include the plots in this paper, we have also numerically studied the case when the interrogating field is a plane wave without decay or a given uniform field. We observed the scheme does not behave well for the uniform field and that although the stability and accuracy of the near field scheme are essentially independent of the plane wave direction, the overall performance 


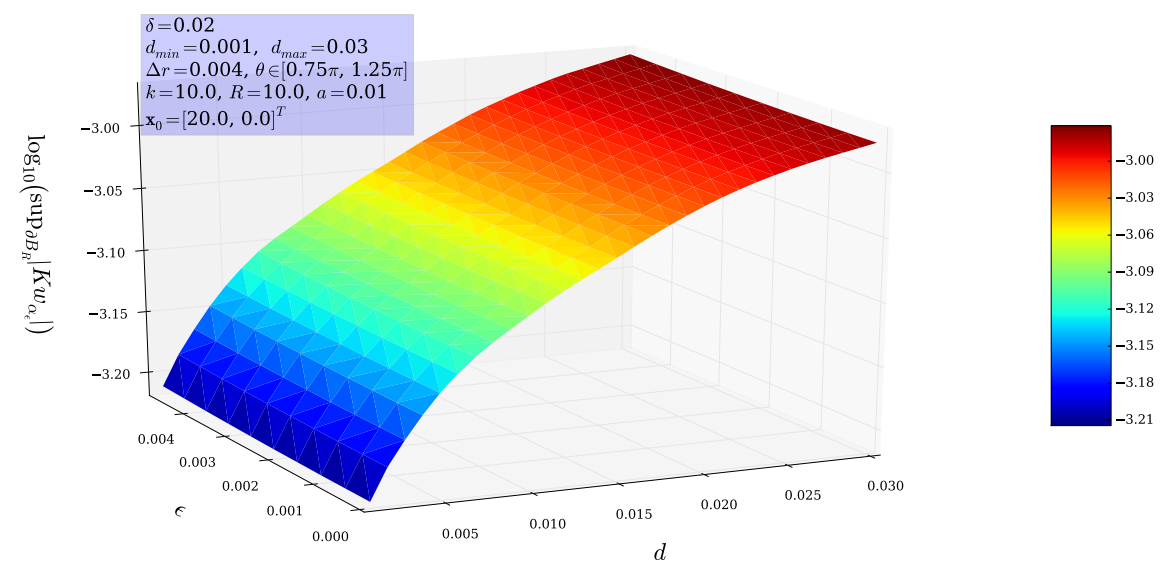

Figure 16: Plot of the far field on $\partial B_{R}$ vs. $d$ and $\epsilon$ for $f_{1}$ a spherical point source at $\mathbf{x}_{0}=[10000,0]^{T}$.
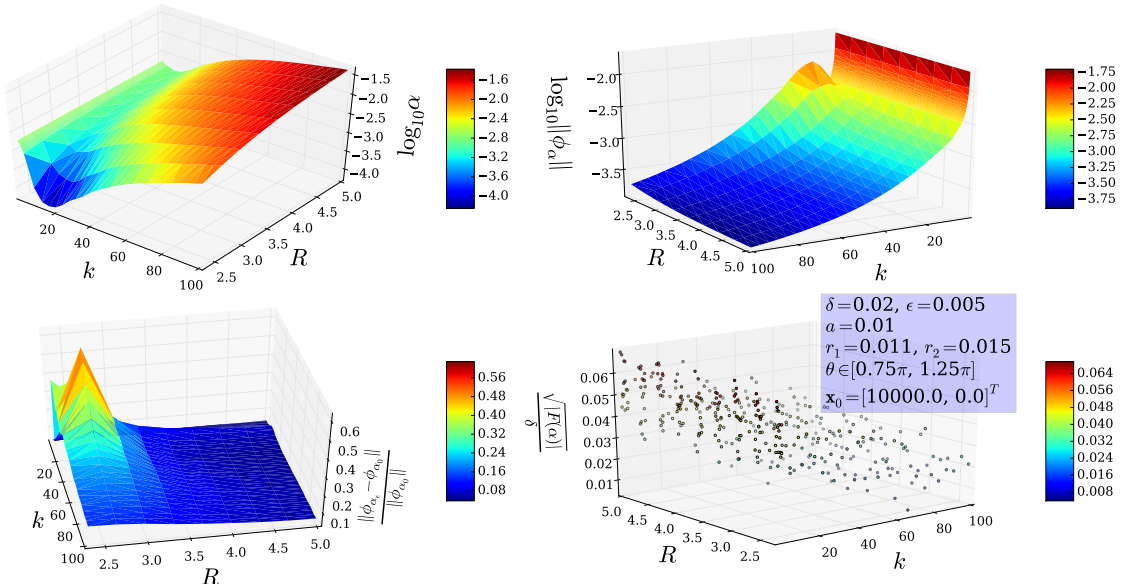

Figure 17: Plot vs. $k$ and $R$ for $f_{1}$ a spherical point source at $\mathbf{x}_{0}=[10000,0]^{T}$.

of the scheme is not as good when compared to the case of an interrogating signal coming from a far field observer presented above. In particular, in the case of a nearfield control region as in, e.g., Figure 13, we observed satisfactory numerical control of a plane wave so long as its direction is not too close to $\pm \pi / 2$, which is related to the shape and orientation of the control region. Moreover, such behaviour seems to become more exaggerated as $k$ increases. Also, for the same settings as in Figure 2 when comparing the case of an interrogating far field point source with an interrogating plane wave, we obtained $5 \%$ versus $8 \%$ stability error and power budget levels of $\approx 10^{-1}$ versus $\approx 10$. We conclude that the scheme performance depends not only on the location of the control region with respect to the source region but also on the amplitude and oscillatory pattern of the incoming field. 
Our analysis makes use of Hypothesis 1 which was suggested by the numerics but which we could not prove at this point. Nevertheless we believe that our discussion presents a strong argument for the characterization of almost nonradiating sources with controllable near fields and for the possibility of stable approximation/cancelling of unwanted interrogating signals.

Currently we are considering the extension of the sensitivity numerical analysis for scalar fields to 3D and the problem of active manipulation for linear arrays and for large elongated antennas. Then, as a next step in our research efforts, we will work on the extension of the stability analysis to the full Maxwell system in free space.

\section{Acknowledgements}

M. Hubenthal was partially supported and D. Onofrei was partially supported by the AFOSR under the 2013 YIP Award FA9550-13-1-0078 and by ONR under the award N00014-15-1-2462.

[1] A. Bakushinsky, A. Goncharsky, Ill-posed problems: theory and applications, volume 301 of Mathematics and its Applications, Kluwer Academic Publishers Group, Dordrecht, 1994. Translated from the Russian by I. V. Kochikov.

[2] P.Y. Chen, C. Argyropoulos, A. Alu, Broadening the cloaking bandwidth with non-foster metasurfaces, Phys. Rev. Lett. 111 (2013) 233001.

[3] D. Colton, R. Kress, Inverse Acoustic and Electromagnetic Scattering Theory, volume 93 of Applied Mathematical Sciences, Springer-Verlag, Berlin, second edition, 1998.

[4] A.J. Devaney, Nonradiating surface sources, J. Opt. Soc. Am. A 21 (2004).

[5] A.J. Devaney, Mathematical foundation of imaging, tomography and wavefield inversion, Cambridge university press, 2012. A Wiley-Interscience Publication.

[6] J. Du, S. Liu, Z. Lin, Broadband optical cloak and illusion created by the low order active sources, Optics Express 20 (2012) 8608-17.

[7] S. Elliot, P. Nelson, Integral equation methods in scattering theory, Electronics and Comm. Engineering Journal 2 (1990) 127-136.

[8] H.W. Engl, M. Hanke, A. Neubauer, Regularization of Inverse Problems, volume 375 of Mathematics and its Applications, Kluwer Academic Publishers Group, Dordrecht, 1996.

[9] C. Fuller, A. von Flotow, Active control of sound and vibration, IEEE 15 (1995) 9-19. 
[10] F. Guevara Vasquez, G.W. Milton, D. Onofrei, Mathematical analysis of the active exterior cloak for $2 \mathrm{~d}$ quasistatic electromagnetics, Analysis and Mathematical Physicsn 2 (????) 231-246.

[11] F. Guevara Vasquez, G.W. Milton, D. Onofrei, Active exterior cloaking for the 2d laplace and helmholtz equations, Physical Review Letters 103 (2009) 073901+.

[12] F. Guevara Vasquez, G.W. Milton, D. Onofrei, Broadband exterior cloaking, Opt. Express 17 (2009) 14800-14805.

[13] F. Guevara Vasquez, G.W. Milton, D. Onofrei, Exterior cloaking with active sources in two dimensional acoustics, Wave Motion 48 (2011) 515-524. Special Issue on Cloaking of Wave Motion.

[14] F. Guevara Vasquez, G.W. Milton, D. Onofrei, P. Seppecher, Transformation elastodynamics and active exterior acoustic cloaking, in: R.V. Craster, S. Guenneau (Eds.), Acoustic Metamaterials, volume 166 of Springer Series in Materials Science, Springer Netherlands, 2013, pp. 289-318.

[15] D. Guicking, Active Control of Sound and Vibration History Fundamentals State of the Art, Festschrift DPI, Herausgeber (ed.), Universitatsverlag Gottingen, 2007.

[16] T. Kato, Perturbation Theory for Linear Operators, Classics in Mathematics, Springer-Verlag, Berlin, 1995. Reprint of the 1980 edition.

[17] A. Kirsch, An introduction to the mathematical theory of inverse problems, volume 120 of Applied Mathematical Sciences, Springer, New York, second edition, 2011.

[18] R. Kress, Linear Integral Equations, volume 82 of Applied Mathematical Sciences, Springer, New York, third edition, 2014.

[19] J. Loncaric, V. Ryaben'kii, S. Tsynkov, Active Shielding and Control of Environmental Noise, Technical Report, Institute for Computer Applications in Science and Engineering (ICASE), 2000.

[20] J. Loncaric, S. Tsynkov, Quadratic optimization in the problems of active control of sound, Applied Numerical Mathematics 52 (2005) 381-400.

[21] P. Lueg, Process of silencing sound oscillations, 1936. US Patent 2,043,416.

[22] Q. Ma, Z.L. Mei, S.K. Zhu, T.Y. Jin, T.J. Cui, Experiments on active cloaking and illusion for laplace equation, Phys. Rev. Lett. 111 (2013) 173901.

[23] E. Marengo, A. Devaney, The inverse source problem of electromagnetics: linear inversion formulation and minimum energy solution, Antennas and Propagation, IEEE Transactions on 47 (1999) 410-412. 
[24] E. Marengo, A. Devaney, G. F.K., Inverse source problem with reactive power constraint, Antennas and Propagation, IEEE Transactions on 52 (2004) 1586-1595.

[25] E. Marengo, R. Ziolkowski, Nonradiating and minimum energy sources and their fields: Generalized source inversion theory and applications, Antennas and Propagation, IEEE Transactions on 48 (2000).

[26] D.A.B. Miller, On perfect cloaking, Opt. Express 14 (2006) 12457-12466.

[27] A.N. Norris, F.A. Amirkulova, W.J. Parnell, Source amplitudes for active exterior cloaking, Inverse Problems 28 (2012) 105002, 20.

[28] H.F. Olson, E.G. May, Electronic sound absorber, The Journal of the Acoustical Society of America 25 (1953) 829-829.

[29] D. Onofrei, On the active manipulation of fields and applications: I. The quasistatic case, Inverse Problems 28 (2012) 105009, 15.

[30] D. Onofrei, Active manipulation of fields modeled by the Helmholtz equation, J. Integral Equations Appl. 26 (2014) 553-579.

[31] N. Peake, D. Crighton, Active control of sound, Annual Review of Fluid Mechanics 32 (2000) 137-164.

[32] A. Peterson, S. Tsynkov, Active control of sound for composite regions, SIAM J. Appl. Math. 67 (2007) 1582-1609.

[33] B. Popa, D. Shinde, A. Konneker, S. Cummer, Active acoustic metamaterials reconfigurable in real time, Phys. Rev. B 91 (2015).

[34] M. Selvanayagam, G. Eleftheriades, An active electromagnetic cloak using the equivalence principle, Antennas and Wireless Propagation Letters, IEEE 11 (2012) 1226-1229.

[35] M. Selvanayagam, G.V. Eleftheriades, Experimental demonstration of active electromagnetic cloaking, Phys. Rev. X 3 (2013) 041011.

[36] J. Soric, P. Chen, A. Kerkhoff, D. Rainwater, K. Melin, A. Alu, Simulation analysis of an active cancellation stealth system, Optik - International Journal for Light and Electron Optics (2014) 52735277.

[37] H. Zheng, J. Xiao, Y. Lai, C. Chan, Exterior optical cloaking and illusions by using active sources: A boundary element perspective, Phys. Rev. B 81 (2010) 195116. 